\title{
Magnetic catalysis in flavored ABJM
}

\author{
Niko Jokela, ${ }^{a}$ Alfonso V. Ramallo ${ }^{a}$ and Dimitrios Zoakos ${ }^{b}$ \\ ${ }^{a}$ Departamento de Física de Partículas, Universidade de Santiago de Compostela and Instituto \\ Galego de Física de Altas Enerxías (IGFAE), E-15782 Santiago de Compostela, Spain \\ ${ }^{b}$ Centro de Física do Porto and Departamento de Fúsica e Astronomia, Faculdade de Ciências da \\ Universidade do Porto, Rua do Campo Alegre 687, 4169-007 Porto, Portugal \\ E-mail: niko.jokela@usc.es, alfonso@fpaxp1.usc.es, \\ dimitrios.zoakos@fc.up.pt
}

ABSTRACT: We study the magnetic catalysis of chiral symmetry breaking in the ABJM Chern-Simons matter theory with unquenched flavors in the Veneziano limit. We consider a magnetized D6-brane probe in the background of a flavored black hole which includes the backreaction of massless smeared flavors in the ABJM geometry. We find a holographic realization for the running of the quark mass due to the dynamical flavors. We compute several thermodynamic quantities of the brane probe and analyze the effects of the dynamical quarks on the fundamental condensate and on the phase diagram of the model. The dynamical flavors have an interesting effect on the magnetic catalysis. At zero temperature and fixed magnetic field, the magnetic catalysis is suppressed for small bare quark masses whereas it is enhanced for large values of the mass. When the temperature is non-zero there is a critical magnetic field, above which the magnetic catalysis takes place. This critical magnetic field decreases with the number of flavors, which we interpret as an enhancement of the catalysis.

KEYwords: D-branes, AdS-CFT Correspondence

ARXIV EPRINT: 1311.6265 


\section{Contents}

1 Introduction 1

2 Holographic model $\quad 3$

2.1 Background metric 3

2.2 D6-brane action 5

$\begin{array}{lll}2.3 & \text { Parameterization at non-zero temperature } & 6\end{array}$

$\begin{array}{lll}2.4 & \text { Parameterization at zero temperature } & 7\end{array}$

2.5 Running mass and condensate 8

3 Some properties of the dual matter $\quad 11$

$\begin{array}{ll}3.1 \text { Thermodynamic functions } & 11\end{array}$

3.2 Massless embeddings 13

$\begin{array}{ll}\text { 3.2.1 Small magnetic field } & 16\end{array}$

$\begin{array}{lll}3.3 & \text { Approximate expressions for large mass } & 16\end{array}$

$\begin{array}{lll}\text { 3.3.1 Zero temperature limit } & 18\end{array}$

4 Magnetic catalysis $\quad 18$

$\begin{array}{lll}4.1 \text { Zero temperature } & 19\end{array}$

$\begin{array}{lll}\text { 4.1.1 Zero bare mass } & 19\end{array}$

4.1.2 Non-zero bare mass 20

4.2 Non-zero temperature 22

$\begin{array}{lll}4.2 .1 & \text { Zero bare mass } & 22\end{array}$

4.2.2 Non-zero bare mass 22

5 Conclusions 23

$\begin{array}{ll}\text { A Zero temperature dictionary } & 25\end{array}$

\section{Introduction}

The dynamics of gauge theories in external electromagnetic fields has revealed a rich structure of new phenomena (see [1] for a recent review). One of these effects is the spontaneous symmetry breaking of chiral symmetry induced by a magnetic field, which is known as magnetic catalysis [2-7]. It can be understood as due to the fermionic pairing and the effective dimensional reduction which take place in the Landau levels. In strongly interacting systems the holographic duality [8] can be used to study this phenomenon $[9]$ (see $[10,11]$ for reviews and further references). The general objective of these holographic studies is to uncover new physical effects of universal nature that are difficult to discover by using more conventional approaches. 
In the holographic approach, the matter fields transforming in the fundamental representation of the gauge group are introduced by adding flavor D-branes to the gravity dual. If these flavor branes are treated as probes and their backreaction on the geometry is neglected, we are in the so-called quenched approximation, which corresponds to discarding quark loops on the field theory side. The magnetic field needed for the catalysis is introduced as a worldvolume gauge field on the D-brane. From the study of the embeddings of the probe one can extract the $\bar{q} q$ condensate as a function of the quark mass and verify the breaking of chiral symmetry induced by the magnetic field.

To go beyond the probe approximation and to study the effects of quark loops in the holographic approach one has to construct new supergravity duals which include the backreaction of the flavor brane sources on the geometry. Finding these unquenched backgrounds is a very difficult problem which can be simplified by considering a continuous distribution of flavor branes (see [12] for a review of this smearing technique). In [13, 14] the magnetic catalysis for the D3-D7 system with unquenched smeared flavor branes was studied and the effects of dynamical flavors on the magnetic catalysis were analyzed.

In this paper we address the problem of the magnetic catalysis with unquenched flavors in the ABJM theory [15]. The unflavored version of the ABJM model is a $(2+1)$ dimensional Chern-Simons matter theory with $\mathcal{N}=6$ supersymmetry, whose gauge group is $\mathrm{U}(N) \times \mathrm{U}(N)$, with Chern-Simons levels $k$ and $-k$. It also contains bifundamental matter fields. When the two parameters $N$ and $k$ are large, the ABJM theory can be holographically described by the ten-dimensional geometry $A d S_{4} \times \mathbb{C P}^{3}$ with fluxes. One can naturally add flavor D6-branes extended along the $A d S_{4}$ and wrapping an $\mathbb{R P}^{3}$ submanifold of the internal $\mathbb{C P}^{3}[16,17]$. The smeared unquenched background for a large number $N_{f}$ of massless flavors has been constructed in [18]. These results were generalized in [19] to non-zero temperature and in [20] to massive flavors. The main advantage of the ABJM case as compared to other holographic setups is that the corresponding flavored backgrounds have a good UV behavior without the pathologies present in other unquenched backgrounds (such as, for example, the Landau pole singularity of the D3-D7 case). Moreover, in the case of massless flavors the geometry is known analytically and is of the form $A d S_{B H_{4}} \times \mathcal{M}_{6}$, where $A d S_{B H_{4}}$ is a black hole in $A d S_{4}$ and $\mathcal{M}_{6}$ is a squashed version of $\mathbb{C P}^{3}$. This simplicity will allow us to obtain a holographic realization of the Callan-Symanzik equation for the running of the quark mass due to the anomalous dimension generated by the unquenched flavors.

We will carry out our analysis by considering a magnetized D6-brane probe in the geometry $[18,19]$ dual to the ABJM theory with unquenched massless flavors (i.e., dynamical sea quarks), corresponding to the backreaction of a large number $N_{f}$ of flavor D6-branes with no magnetic field. We are thus neglecting the influence of the magnetic field on the sea quarks. To take this effect into account we would have to find the backreaction to magnetized flavor D6-branes, which is an involved problem beyond the scope of this work. We will study the system both at zero and non-zero temperature. In both cases we will be able to study the influence of the dynamical sea quarks at fully non-linear order in $N_{f}$.

The rest of this paper is organized as follows. In section 2 we introduce our holographic model. We review the background of $[18,19]$, study the action of the probe in several 
coordinate systems and establish the dictionary to relate the holographic parameters to the physical mass and condensate. In section 3 we obtain the different thermodynamic properties of the magnetized brane and we find analytic results in some particular limiting cases. Section 4 is devoted to the study of the phase diagram and of the magnetic catalysis of chiral symmetry breaking. Finally, in section 5 we summarize our results and discuss some possible research directions for the future. Appendix A contains the derivation of the holographic dictionary for the condensate at zero temperature.

\section{Holographic model}

In this section we will recall the background of type IIA supergravity dual to unquenched massless flavors in the ABJM Chern-Simons matter theory at non-zero temperature. This background was obtained $[18,19]$ by including the backreaction of $N_{f}$ flavor D6-branes, which are continuously distributed in the internal space in such a way that the system preserves $\mathcal{N}=1$ supersymmetry at zero temperature. This smearing procedure is a holographic implementation of the so-called Veneziano limit [21], in which both $N$ and $N_{f}$ are large. As the smeared flavor branes are not coincident the flavor symmetry is $\mathrm{U}(1)^{N_{f}}$ rather than $\mathrm{U}\left(N_{f}\right)$.

To study magnetic catalysis in this gravity dual with unquenched flavors, we will add an additional flavor D6-brane probe with a magnetic field in its worldvolume. We will obtain the action of this probe and introduce various systems of coordinates which are convenient to describe the embeddings of the brane, both at zero and non-zero temperature.

\subsection{Background metric}

Our model consists of a probe D6-brane in the smeared flavored ABJM background of [18, 19], oriented such that their intersection is $(2+1)$-dimensional. We will begin by laying out our conventions and reviewing the background geometry. The metric of the background is $[18,19]$

$$
d s_{10}^{2}=L^{2}\left(-h r^{2} d t^{2}+r^{2}\left(d x^{2}+d y^{2}\right)+\frac{d r^{2}}{h r^{2}}\right)+\frac{L^{2}}{b^{2}}\left(q d s_{\mathbb{S}^{4}}^{2}+\left(E^{1}\right)^{2}+\left(E^{2}\right)^{2}\right)
$$

where $L$ is a constant radius and the blackening factor is $h(r)=1-\frac{r_{h}^{3}}{r^{3}}$, with $r_{h}$ constant. In our conventions all coordinates are dimensionless and $L$ has dimension of length. The Bekenstein-Hawking temperature $T$ of the black hole is related to $r_{h}$ as $T=\frac{3 r_{h}}{4 \pi}$. Notice that $T$ is dimensionless (the physical temperature is $T / \sqrt{\alpha^{\prime}}$ ). The internal metric in (2.1) is a deformation of the Fubini-Study metric of $\mathbb{C P}^{3}$, represented as an $\mathbb{S}^{2}$-bundle over $\mathbb{S}^{4}$. This deformation is generated by the backreaction of the massless flavors and introduces a relative squashing $q$ between the $\mathbb{S}^{2}$ fiber, corresponding to the two one-forms $E^{1}$ and $E^{2}$, and the $\mathbb{S}^{4}$ base. We write the metric on the four-sphere in (2.1) as

$$
d s_{\mathbb{S}^{4}}^{2}=\frac{4}{\left(1+\xi^{2}\right)^{2}}\left[d \xi^{2}+\xi^{2} \sum_{i=1}^{3}\left(\omega^{i}\right)^{2}\right],
$$


where $0 \leq \xi<\infty$ is a non-compact coordinate and the $\omega^{i}$ are $\mathrm{SU}(2)$ left-invariant oneforms satisfying $d \omega^{i}=\frac{1}{2} \epsilon_{i j k} \omega^{j} \wedge \omega^{k}$. The $\mathbb{S}^{2}$ will be represented by the ordinary polar coordinates $0 \leq \theta<\pi$ and $0 \leq \varphi<2 \pi$, in terms of which $E^{1}$ and $E^{2}$ can be written as

$$
\begin{aligned}
& E^{1}=d \theta+\frac{\xi^{2}}{1+\xi^{2}}\left(\sin \varphi \omega^{1}-\cos \varphi \omega^{2}\right) \\
& E^{2}=\sin \theta\left(d \varphi-\frac{\xi^{2}}{1+\xi^{2}} \omega^{3}\right)+\frac{\xi^{2}}{1+\xi^{2}} \cos \theta\left(\cos \varphi \omega^{1}+\sin \varphi \omega^{2}\right)
\end{aligned}
$$

The metric (2.1) has two parameters $q$ and $b$ which deserve pronunciation. The parameter $q$ is a constant squashing factor of the internal $\mathbb{C P}^{3}$ sub-manifold, whereas $b$ represents the relative squashing between the internal space and the $A d S_{B H_{4}}$ part of the metric. The explicit expressions for the factors $q$ and $b$ of the smeared solution of $[18,19]$ are:

$$
\begin{aligned}
q & =3+\frac{3}{2} \hat{\epsilon}-2 \sqrt{1+\hat{\epsilon}+\frac{9}{16} \hat{\epsilon}^{2}} \\
b & =\frac{2 q}{q+1},
\end{aligned}
$$

where $\hat{\epsilon}$ is the flavor deformation parameter, which depends on the number of flavors $N_{f}$ and colors $N$, as well as the 't Hooft coupling $\lambda=N / k$, via

$$
\hat{\epsilon}=\frac{3 N_{f}}{4 k}=\frac{3}{4} \frac{N_{f}}{N} \lambda .
$$

The radius $L$ in (2.1) is also modified by the backreaction of the flavors. Indeed, it can be written as [18]

$$
L^{2}=\pi \sqrt{2 \lambda} \sigma \alpha^{\prime},
$$

where $\sigma$ is the so-called screening function, which determines the correction of the radius with respect to the unflavored case and is given by the following function of the deformation parameter

$$
\sigma \equiv \sqrt{\frac{2-q}{q[q+(1+\hat{\epsilon})(q-1)]}} b^{2}=\frac{1}{4} \frac{q^{\frac{3}{2}}(2-q)^{\frac{1}{2}}(1+\hat{\epsilon}+q)^{2}}{[q+(1+\hat{\epsilon})(q-1)]^{\frac{5}{2}}} .
$$

We note that most of the equations that we will manipulate in this paper only depend on $b$, in which case it suffices to keep in mind that $b$ is monotonously increasing between 1 and $5 / 4$ as one dials $\hat{\epsilon}=0$ to $\infty$. Moreover, $\sigma=1$ for $\hat{\epsilon}=0$ and it vanishes as $1 / \sqrt{\hat{\epsilon}}$ when $\hat{\epsilon}$ is large.

The type IIA supergravity solution of $[18,19]$ also contains a constant dilaton $\phi$, given by

$$
e^{-\phi}=\frac{b}{4} \frac{1+\hat{\epsilon}+q}{2-q} \frac{k}{L} \sqrt{\alpha^{\prime}},
$$

as well as RR forms $F_{2}$ and $F_{4}$. In this paper we will only need the seven-form potential $C_{7}$ of $F_{8}=-* F_{2}$. To avoid unnecessary notation, we shall only present its pullback in the subsection to follow. 


\subsection{D6-brane action}

Next we will add a probe D6-brane in this background, extended along the Minkowski and radial coordinates and wrapping a three cycle $\mathcal{C}_{3} \simeq \mathbb{R P}^{3}$ inside the internal manifold. The cycle $\mathcal{C}_{3}$ extends along two directions of the $\mathbb{S}^{4}$ and one direction of the $\mathbb{S}^{2}$ fiber. It can be characterized by requiring that the pullbacks of two of the one-forms $\omega^{i}$ vanish (say, $\omega^{1}$ and $\omega^{2}$ ) and that the angle $\theta$ of the $\mathbb{S}^{2}$ is a function of the radial variable. By a suitable choice of coordinates, ${ }^{1}$ the induced metric on the D6-brane worldvolume can be written as

$$
\begin{aligned}
d \hat{s}_{7}^{2}= & -L^{2} r^{2} d t^{2}+L^{2} r^{2}\left[\left(d x^{1}\right)^{2}+\left(d x^{2}\right)^{2}\right]+\frac{L^{2}}{r^{2}}\left[1+\frac{r^{2}}{b^{2}} \dot{\theta}^{2}\right] d r^{2} \\
& +\frac{L^{2}}{b^{2}}\left[q d \alpha^{2}+q \sin ^{2} \alpha d \beta^{2}+\sin ^{2} \theta(d \psi+\cos \alpha d \beta)^{2}\right],
\end{aligned}
$$

where $\dot{\theta}=d \theta / d r$ and $0 \leq \alpha<\pi, 0 \leq \beta, \psi<2 \pi$.

The D6-brane action has two contributions. As usual there is the Dirac-Born-Infeld term, but we also have a Chern-Simons term due to the pullback of the RR seven-form potential

$$
S_{D 6}=-T_{D 6} e^{-\phi} \int d^{7} \zeta \sqrt{-\operatorname{det}\left(g_{7}+F\right)}+T_{D 6} \int \hat{C}_{7}
$$

where the $\zeta$ 's are the coordinates of the induced metric and $F=d A$ is the strength of the worldvolume gauge field. The explicit form of the the pullback of $C_{7}$ is [19]

$$
\hat{C}_{7}=\frac{L^{7} q}{b^{3}} e^{-\phi} d^{3} x \wedge\left[\frac{h r^{3}}{b} \sin \theta \cos \theta \dot{\theta}+r^{2} \sin ^{2} \theta+L_{2}(r)\right] \wedge d r \wedge \Xi_{3},
$$

where $\Xi_{3}=\sin \alpha d \alpha \wedge d \beta \wedge d \psi$ and $\int d r L_{2}(r)=\frac{r_{h}^{3}}{4 b}$. To write $C_{7}$ we have chosen a particular gauge which leads to a finite renormalized action with consistent thermodynamics. In this paper we will consider a background magnetic field described by a spatial component of the D6-brane gauge field:

$$
A_{x^{2}}=x^{1} L^{2} B \text {. }
$$

In our conventions, the quantity $B$, as well as $x^{1}$, is dimensionless. Notice also that the physical magnetic field is related to $B$ as

$$
B_{\text {phys }}=\frac{L^{2} B}{\alpha^{\prime 2}}=\frac{\pi \sqrt{2 \lambda} \sigma B}{\alpha^{\prime}} .
$$

A straightforward computation for the full action yields:

$$
S=-\mathcal{N} \int d^{3} x\left\{\frac{4 b}{r_{h}^{3}} \int d r r^{2} \sin \theta\left(\sqrt{1+\frac{B^{2}}{r^{4}}} \sqrt{1+h\left(\frac{r}{b}\right)^{2} \dot{\theta}^{2}}-\sin \theta-h \frac{r}{b} \cos \theta \dot{\theta}\right)-1\right\},
$$

where the prefactor is

$$
\mathcal{N}=\frac{2 \pi^{2} r_{h}^{3} L^{7} q}{b^{4}} T_{D 6} e^{-\phi}=\frac{2 \sqrt{2} \pi^{2}(2-b) b \sigma}{27} N \sqrt{\lambda} T^{3} .
$$

\footnotetext{
${ }^{1}$ Let us require the pullbacks $\hat{\omega}^{1}=\hat{\omega}^{2}=0$ and parameterize $\hat{\omega}^{3}=d \hat{\psi}$. Then, $\alpha, \beta$, and $\psi$ are defined as: $\xi=: \tan \left(\frac{\alpha}{2}\right), \beta:=\frac{\hat{\psi}}{2}$, and $\psi:=\varphi-\frac{\hat{\psi}}{2}$.
} 
For later use we also define:

$$
\mathcal{N}_{r}=\frac{4 b}{r_{h}^{3}} \mathcal{N}=\frac{(2-b) b^{2} \sigma}{4 \sqrt{2} \pi} \frac{N^{3 / 2}}{\sqrt{k}} .
$$

The equation of motion for the embedding scalar is thus,

$$
\partial_{r}\left(g\left(\frac{r}{b}\right)^{2}\left(1+\frac{B^{2}}{r^{4}}\right) \dot{\theta}\right)=r^{2}\left(\frac{3}{2 b}-1+\frac{h r^{2}}{2 g}\right) \sin 2 \theta,
$$

where we have defined

$$
g=\frac{h r^{2} \sin \theta}{\sqrt{1+\frac{B^{2}}{r^{4}}} \sqrt{1+h\left(\frac{r}{b}\right)^{2} \dot{\theta}^{2}}} .
$$

The above equation of motion has generically two kinds of solutions. The first kind are embeddings that penetrate the black hole horizon, those we shall call black hole (BH) embeddings. The other kind are Minkowski (MN) embeddings, which terminate smoothly above the horizon at some $r_{0}>r_{h}$. Examples of both kind are the following. Clearly, the equation of motion is satisfied with trivial constant angle BH embeddings $\theta=0, \pi / 2$. The equation of motion possesses a supersymmetric MN solution $\cos \theta(r)=\left(\frac{r_{0}}{r}\right)^{b}$ at $r_{h}=0$ and $B=0$. Away from zero temperature and vanishing magnetic field, this solution has to be analyzed numerically. Our focus in this article is to study how these two types of solutions map out the phase space as both the $T$ and $B$ are dialed, and the interesting effects from the variation of the number of background flavors (essentially $b$ ). Before we will get absorbed in analyzing several aspects of the system, we wish to introduce new parameterizations better suited for the analyses.

\subsection{Parameterization at non-zero temperature}

It is useful to introduce another parameterization as discussed in [19]. Let us introduce a system with isotropic Cartesian-like coordinates

$$
\begin{aligned}
R & =u \cos \theta \\
\rho & =u \sin \theta,
\end{aligned}
$$

where the new radial coordinate $u$ is related to the old one as

$$
u^{\frac{3}{2 b}}=\left(\frac{r}{r_{h}}\right)^{\frac{3}{2}}+\sqrt{\left(\frac{r}{r_{h}}\right)^{3}-1} .
$$

We also define the functions $f$ and $\tilde{f}$ as

$$
\begin{aligned}
& f=1-u^{-3 / b} \\
& \tilde{f}=1+u^{-3 / b} .
\end{aligned}
$$

We also rescale the magnetic field as follows:

$$
\hat{B}=2^{4 / 3} \frac{B}{r_{h}^{2}} .
$$


After these mappings the action becomes

$$
\begin{aligned}
S=-\mathcal{N} \int d^{3} x\{ & \int d \rho \rho f \tilde{f} u^{3 / b-2}\left(\sqrt{1+\frac{\hat{B}^{2}}{\tilde{f}^{8 / 3} u^{4 / b}}} \sqrt{1+R^{\prime 2}}-1\right. \\
& \left.\left.+\left(\frac{f}{\tilde{f}}-1\right) \frac{R}{u^{2}}\left(\rho R^{\prime}-1\right)\right)-1\right\},
\end{aligned}
$$

where it is understood that $u=\sqrt{\rho^{2}+R^{2}}$.

A generic solution to the equation of motion following from the action (2.27), behaves close to the boundary as:

$$
R=m+\frac{c}{\rho^{3 / b-2}}+\ldots, \rho \rightarrow \infty,
$$

where $m$ is related to the quark mass and $c$ is proportional to the vacuum expectation value $\langle\bar{\psi} \psi\rangle$ (see below).

\subsection{Parameterization at zero temperature}

At zero temperature we also make use of the Cartesian-like coordinates as in (2.21) and (2.22), but with

$$
u=r^{b}
$$

The action (2.16) maps to

$$
S=-\frac{\mathcal{N}_{r}}{b} \int d \rho \rho u^{3 / b-2}\left\{\sqrt{1+\frac{B^{2}}{u^{4 / b}}} \sqrt{1+R^{\prime 2}}-1\right\},
$$

where it is understood that $u=\sqrt{\rho^{2}+R^{2}}$. We can scale out the $B$ as follows:

$$
\begin{aligned}
u^{4 / b} & =B^{2} \tilde{u}^{4 / b} \rightarrow u=B^{b / 2} \tilde{u} \\
R & =B^{b / 2} \tilde{R} \\
\rho & =B^{b / 2} \tilde{\rho} .
\end{aligned}
$$

This leads us to

$$
S=-\frac{B^{3 / 2}}{b} \mathcal{N}_{r} \int d \tilde{\rho} \tilde{\rho} \tilde{u}^{3 / b-2}\left\{\sqrt{1+\frac{1}{\tilde{u}^{4 / b}}} \sqrt{1+\tilde{R}^{\prime 2}}-1\right\}
$$

and to the following asymptotic behavior of the embedding function

$$
R \sim m_{0}+\frac{c_{0}}{\rho^{3 / b-2}} \rightarrow \tilde{R} \sim \tilde{m}_{0}+\frac{\tilde{c}_{0}}{\tilde{\rho}^{3 / b-2}},
$$

where we defined the dimensionless quantities $\tilde{m}_{0}$ and $\tilde{c}_{0}$ as:

$$
\begin{aligned}
\tilde{m}_{0} & \equiv B^{-b / 2} m_{0} \\
\tilde{c}_{0} & \equiv B^{(b-3) / 2} c_{0} .
\end{aligned}
$$

The parameters $m_{0}$ and $c_{0}$ can be related to the quark mass $m_{q}$ and the quark condensate at zero temperature (denoted by $\left\langle\mathcal{O}_{q}\right\rangle_{0}$ ). The corresponding relation is worked out in the next section and in appendix A. 


\subsection{Running mass and condensate}

The asymptotic value of the embedding function $R$ should be related to the quark mass. To find the precise relation we will consider a fundamental string stretched in the $R$ direction and ending on the flavor brane. The quark mass is just the Nambu-Goto action of the string per unit time. While carrying out this computation we should take into account that we are dealing with a theory with unquenched quarks in which the quark mass $m_{q}$ acquires an anomalous dimension $\gamma_{m}$ and $m_{q}$ therefore runs with the scale according to the corresponding Callan-Symanzik equation. In our holographic setup the value of $\gamma_{m}$ was found in $[18,19]$ and is simply related to the squashing parameter $b$ :

$$
\gamma_{m}=b-1 \text {. }
$$

In order to find the scale dependence of $m_{q}$, we consider a fundamental string located at the point $\rho=\rho_{*}$. We will start by considering the zero temperature case. Notice that $\rho$ is the holographic coordinate in our setup and, therefore, it is natural to think that the value of $\rho_{*}$ determines the energy scale. The induced metric on a string worldsheet extended in $(t, R)$ at $\rho=\rho_{*}$ when $T=0$ is given by:

$$
d s_{2}^{2}=-L^{2}\left[R^{2}+\rho_{*}^{2}\right]^{\frac{1}{b}} d t^{2}+\frac{L^{2}}{b^{2}} \frac{d R^{2}}{R^{2}+\rho_{*}^{2}} .
$$

The running quark mass at zero temperature is then defined as:

$$
\begin{aligned}
m_{q} & =\frac{1}{2 \pi\left(\alpha^{\prime}\right)^{\frac{3}{2}}} \int_{0}^{m_{0}} \sqrt{-\operatorname{det} g_{2}} d R=\sqrt{\frac{\lambda}{2}} \frac{\sigma}{b \sqrt{\alpha^{\prime}}} \int_{0}^{m_{0}}\left[R^{2}+\rho_{*}^{2}\right]^{\frac{1}{2 b}-\frac{1}{2}} d R \\
& =\sqrt{\frac{\lambda}{2}} \frac{\sigma}{b \sqrt{\alpha^{\prime}}} m_{0} \rho_{*}^{\frac{1}{b}-1}{ }_{2} F_{1}\left(\frac{1}{2}, \frac{\gamma_{m}}{2 b} ; \frac{3}{2} ;-\frac{m_{0}^{2}}{\rho_{*}^{2}}\right) .
\end{aligned}
$$

Notice that, in the unflavored case $b=1, \gamma_{m}=0$ and the effective mass $m_{q}$ is independent of the scale parameter $\rho_{*}$, as it should. To determine the precise relation between $\rho_{*}$ and the energy scale $\Lambda$, let us consider the relation (2.29) between the coordinate $u$ and the canonical $A d S_{4}$ radial coordinate $r$. Taking into account that $u \approx \rho$ in the UV, it is natural to identify $r$ with the energy scale and define $\Lambda$ as:

$$
\Lambda \equiv \rho_{*}^{\frac{1}{b}} .
$$

The dependence of $m_{q}$ on $\Lambda$ can be straightforwardly inferred from (2.40). Moreover, from the integral representation in (2.40) we can readily obtain an evolution equation for $m_{q}$

$$
\frac{\partial m_{q}}{\partial \log \Lambda}=m_{q}-\frac{\sigma}{\sqrt{\alpha^{\prime}}} \sqrt{\frac{\lambda}{2}} \frac{m_{0}}{\left(\Lambda^{2 b}+m_{0}^{2}\right)^{\frac{\gamma m}{2 b}}} .
$$

Clearly, the second term in (2.42) incorporates the flavor effects on the running of $m_{q}$. In the UV regime of large $\Lambda$ we can just neglect $m_{0}^{2}$ in the denominator of (2.42). The solution of this UV equation can be obtained directly or by taking the large $\Lambda$ limit of (2.40). We get

$$
\frac{m_{q} \sqrt{\alpha^{\prime}}}{\sqrt{\lambda}} \approx \frac{\sigma}{\sqrt{2} b} m_{0} \Lambda^{-\gamma_{m}},
$$


which shows that in the UV $m_{q}$ and $m_{0}$ are proportional and that the running of $m_{q}$ with the scale $\Lambda$ is controlled by the mass anomalous dimension $\gamma_{m}$. Notice that the UV mass (2.43) satisfies:

$$
\frac{\partial m_{q}}{\partial \log \Lambda}=-\gamma_{m} m_{q}
$$

which is just the Callan-Symanzik equation for the effective mass.

The analysis carried out above for $m_{q}$ is independent of the value of the magnetic field $B$. When $B \neq 0$ it is convenient to write the solution of the evolution equation in terms of the reduced mass parameter $\tilde{m}_{0}$ defined in (2.36). We get:

$$
\frac{m_{q} \sqrt{\alpha^{\prime}}}{\sqrt{\lambda}}=\frac{\sigma}{\sqrt{2} b} B^{\frac{b}{2}} \tilde{m}_{0} \Lambda^{-\gamma_{m}}{ }_{2} F_{1}\left(\frac{1}{2}, \frac{\gamma_{m}}{2 b} ; \frac{3}{2} ;-\frac{B^{b} \tilde{m}_{0}^{2}}{\Lambda^{2\left(1+\gamma_{m}\right)}}\right) .
$$

To find the relation between the parameter $c_{0}$ in (2.35) and the condensate we have to compute the derivative of the free energy with respect to the bare quark mass $\mu_{q}^{0}$, which is the quark mass without the screening effects due to the quark loops. These effects are encoded in the functions $\sigma$ and $b$. By putting $\sigma=b=1$, which corresponds to taking $\hat{\epsilon}=0$, we switch off the dressing due to the dynamical flavors. Accordingly, to get $\mu_{q}^{0}$ in terms of $\tilde{m}_{0}$ we just take $\sigma=b=1$ on the right-hand side of (2.45). We get

$$
\mu_{q}^{0}=\sqrt{\frac{\lambda}{2}} \frac{\sqrt{B} \tilde{m}_{0}}{\sqrt{\alpha^{\prime}}}
$$

Notice that the value of $\tilde{m}_{0}$ does not depend on the magnetic field, which is factorized in the action (2.34). Therefore, the dependence of $\mu_{q}^{0} \sim \sqrt{B}$ on the field $B$ is the same as in the unflavored case, as it should.

The explicit calculation of the vacuum expectation value $\left\langle\mathcal{O}_{q}\right\rangle_{0}$ has been performed in appendix A, with the result:

$$
-\frac{\left\langle\mathcal{O}_{q}\right\rangle_{0} \alpha^{\prime}}{N}=\frac{(3-2 b)(2-b)}{4 \pi} \sigma B^{\frac{\gamma m}{2}} c_{0}=\frac{(3-2 b)(2-b)}{4 \pi} \sigma B \tilde{c}_{0} .
$$

eqs. (2.46) and (2.47) constitute the basic dictionary in our analysis of the chiral symmetry breaking at zero temperature.

For non-zero temperature we shall proceed as in the $T=0$ case. The induced metric for the fundamental string extended in $R$ at $\rho=\rho_{*}$ is now

$$
d s_{2}^{2}=-\frac{L^{2} r_{h}^{2}}{2^{\frac{4}{3}}}\left[R^{2}+\rho_{*}^{2}\right]^{\frac{1}{b}}\left[f_{*}(R)\right]^{2}\left[\tilde{f}_{*}(R)\right]^{-\frac{2}{3}} d t^{2}+\frac{L^{2}}{b^{2}} \frac{d R^{2}}{R^{2}+\rho_{*}^{2}},
$$

where $f_{*}(R)$ and $\tilde{f}_{*}(R)$ are the functions defined in (2.24) and (2.25) at $\rho=\rho_{*}$. Accordingly, the running quark mass at $T \neq 0$ is now given by an integral extended from the horizon (for $\rho_{*}<1$ ) to $R=m$ :

$$
m_{q}^{T}=\sqrt{\frac{\lambda}{2}} \frac{\sigma}{b \sqrt{\alpha^{\prime}}} \frac{r_{h}}{2^{\frac{2}{3}}} \int_{R_{h}}^{m}\left[R^{2}+\rho_{*}^{2}\right]^{\frac{1}{2 b}-\frac{1}{2}} f_{*}(R)\left[\tilde{f}_{*}(R)\right]^{-\frac{1}{3}} d R,
$$


where $R_{h}=\sqrt{1-\rho_{*}^{2}}$ for $\rho_{*}<1$ and $R_{h}=0$ otherwise. We have not been able to integrate analytically this expression for arbitrary values of $\rho_{*}$. In order to relate $\rho_{*}$ with the scale $\Lambda$ we recall that, in the UV, $\rho^{\frac{1}{b}} \approx u^{\frac{1}{b}} \approx 2^{\frac{2}{3}} r / r_{h}$. Therefore, identifying again $r$ with $\Lambda$, we have:

$$
\Lambda=2^{-\frac{2}{3}} r_{h} \rho_{*}^{\frac{1}{b}} .
$$

We readily obtain in the UV domain $(\Lambda \gg 1)$ :

$$
\frac{m_{q}^{T} \sqrt{\alpha^{\prime}}}{\sqrt{\lambda}} \approx \frac{\sigma}{\sqrt{2} b} \frac{r_{h}^{b}}{2^{\frac{2 b}{3}}} m \Lambda^{-\gamma_{m}} .
$$

This UV function $m_{q}^{T}$ also satisfies the Callan-Symanzik equation (2.44). Actually, it is easy to relate in the UV the effective mass $m_{q}^{T}$ to its zero temperature counterpart. In order to establish this connection, let us connect $m_{0}$ and $c_{0}$ with the zero temperature limit of $m$ and $c$. To find these relations we recall that these parameters characterize the leading and subleading UV behaviors of the embedding function. From this observation it is easy to prove that

$$
m^{\frac{1}{b}} \approx 2^{\frac{2}{3}} r_{h}^{-1} m_{0}^{\frac{1}{b}}, \quad c \approx 2^{2-\frac{2 b}{3}} r_{h}^{b-3} c_{0}, \quad(T \rightarrow 0) .
$$

By using the relation between $m$ and $m_{0}$ written in (2.52), we see that $m_{q}$ is just the limit of $m_{q}^{T}$ as $T \rightarrow 0$ :

$$
m_{q}=\lim _{T \rightarrow 0} m_{q}^{T} .
$$

The bare mass at non-zero temperature $\mu_{q}$ is obtained from the unflavored limit of the UV running mass (2.51). We get [19]:

$$
\mu_{q} \sqrt{\alpha^{\prime}}=\frac{2^{\frac{1}{3}} \pi}{3} \sqrt{2 \lambda} T m
$$

The resulting vacuum expectation value at $T \neq 0$ has been obtained in appendix D of [19] and is given by:

$$
-\frac{\left\langle\mathcal{O}_{q}\right\rangle \alpha^{\prime}}{N}=\frac{2^{2 / 3} \pi(3-2 b)(2-b)}{9} \sigma T^{2} c .
$$

The relation between the condensates at non-zero and zero temperature is similar to the one corresponding to the masses. Indeed, by using (2.52) we get that $\left\langle\mathcal{O}_{q}\right\rangle_{0}$ is given by the following zero temperature limit:

$$
\left\langle\mathcal{O}_{q}\right\rangle_{0}=\lim _{T \rightarrow 0}\left[\hat{B}^{\frac{\gamma m}{2}}\left\langle\mathcal{O}_{q}\right\rangle\right]
$$

The relation (2.56) is very natural from the point of view of the renormalization group. Indeed, $\left\langle\mathcal{O}_{q}\right\rangle$ and $\left\langle\mathcal{O}_{q}\right\rangle_{0}$ are dimensionful quantities defined at scales determined by the temperature and the magnetic field, respectively. The quotient $\left\langle\mathcal{O}_{q}\right\rangle /\left\langle\mathcal{O}_{q}\right\rangle_{0}$ should be given by the ratio of these two energy scales (which is basically $\sqrt{\hat{B}}$ ) raised to some power which, following the renormalization group logic, should be the mass anomalous dimension, as in (2.56).

In the rest of this paper, we will use units in which $\alpha^{\prime}=1$. The appropriate power of $\alpha^{\prime}$ can be easily obtained in all expressions by looking at their units. 


\section{Some properties of the dual matter}

We will discuss many of the characteristics of the dual matter as described by the gravitational system. The BH phase describes typical metallic behavior. The phase is nongapped to charged and neutral excitations. For example, the former can be easily verified by the standard DC conductivity calculation [22] in a simple generalization of our model by introducing a non-vanishing charge density on the probe. The MN phase, on the other hand, behaves like an insulator: it is gapped to both neutral and charged excitations; the latter can be checked by the conductivity calculation of [23] and the former by fluctuation analysis. The interplay between these two phases in the presence of a charge density makes an interesting story, which will be addressed in a future work.

In the absence of the magnetic field, the thermodynamic properties of the system were discussed in great detail in [19]. Here we are more interested in the magnetic properties and on the effects that the magnetic field will bear. We will break this narrative in two parts, so that in this section we will constrain ourselves to the case where we have analytic control and in the next section we will confront the numerical side of the story, most relevantly the magnetic catalysis.

\subsection{Thermodynamic functions}

The free energy of the system is obtained from evaluating the Wick rotated on-shell action (2.16). As discussed in [18, 19], the free energy is finite albeit subtle at non-zero temperature; there is no need to invoke holographic renormalization to get rid off infinities. The free energy of the probe is identified with the Euclidean on-shell action $S_{E}$, through the relation $F=T S_{E}$. In the calculation of $S_{E}$ we integrate over both the Euclidean time and the non-compact two-dimensional space. Since the latter integration gives rise to an (infinite) two-dimensional volume $V_{2}$, from now on we divide all the extensive thermodynamic quantities by $V_{2}$ and deal with densities. The free energy density $F$ can be written as

$$
\frac{F}{\mathcal{N}}=\mathcal{G}(m, \hat{B})-1
$$

The explicit expression for the function $\mathcal{G}(m, \hat{B})$ can be obtained from the action of the D6-brane probe. For MN embeddings it is more convenient to use $R(\rho)$ as embedding function. From the expression (2.27) of the action in these variables, $\mathcal{G}(m, \hat{B})$ is given by

$$
\begin{gathered}
\mathcal{G}(m, \hat{B})=\int_{0}^{\infty} d \rho \rho\left[\rho^{2}+R^{2}\right]^{\frac{3}{2 b}-1} f \tilde{f}\left[\sqrt{1+R^{\prime 2}} \sqrt{1+\frac{\hat{B}^{2}}{\tilde{f}^{\frac{8}{3}}}\left[\rho^{2}+R^{2}\right]^{-\frac{2}{b}}}\right. \\
\left.-1+\left(\frac{f}{\tilde{f}}-1\right) \frac{R}{\rho^{2}+R^{2}}\left(\rho R^{\prime}-R\right)\right] .
\end{gathered}
$$

For black hole embeddings it is better to use the $\theta=\theta(r)$ parameterization and represent $\mathcal{G}(m, \hat{B})$ as:

$\mathcal{G}(m, \hat{B})=\frac{4 b}{r_{h}^{3}} \int_{r_{h}}^{\infty} d r r^{2} \sin ^{2} \theta\left[\sqrt{1+\left(\frac{r_{h}}{\left.2^{\frac{2}{3}}\right)^{4}} \hat{B}^{2}\right.} \sqrt{1+h\left(\frac{r}{b}\right)^{2} \dot{\theta}^{2}}-\sin \theta-h \frac{r}{b} \cos \theta \dot{\theta}\right]$. 
The fact that the system under study is defined at a fixed temperature and magnetic field implies that the appropriate thermodynamic potential is

$$
d F=-s d T-\mathcal{M} d B
$$

where $s$ is the entropy density and $\mathcal{M}$ is the magnetization of the system. Following (3.4), the entropy density $s$ is given by the following expression

$$
s=-\left(\frac{\partial F}{\partial T}\right)_{B}=-\frac{\mathcal{N}}{T}\left[\frac{3 F}{\mathcal{N}}+T\left(\frac{\partial \mathcal{G}}{\partial m}\right)_{\hat{B}}\left(\frac{\partial m}{\partial T}\right)_{B}+T\left(\frac{\partial \mathcal{G}}{\partial \hat{B}}\right)_{m}\left(\frac{\partial \hat{B}}{\partial T}\right)_{B}\right] .
$$

Let us compute the different derivatives on the right-hand side of (3.5). First of all we use that [19]

$$
\frac{\partial \mathcal{G}}{\partial m}=\frac{2 b-3}{b} c
$$

and that $T \partial m / \partial T=-b m$, as follows from (2.51) when $m_{q}^{T}$ and $\Lambda$ are fixed. Moreover, we define the function $\mathcal{J}(m, \hat{B})$ as

$$
\mathcal{J}(m, \hat{B}) \equiv \frac{1}{\hat{B}} \frac{\partial \mathcal{G}(m, \hat{B})}{\partial \hat{B}} .
$$

Then, taking into account the $\hat{B} \propto T^{-2}$ temperature dependence of the rescaled magnetic field in (2.26), we get:

$$
T \frac{s}{\mathcal{N}}=3-3 \mathcal{G}(m, \hat{B})+2 \hat{B}^{2} \mathcal{J}(m, \hat{B})-(3-2 b) c m .
$$

For Minkowski embeddings, $\mathcal{J}(m, \hat{B})$ is explicitly given by the following integral:

$$
\mathcal{J}(m, \hat{B}) \equiv \int_{0}^{\infty} d \rho\left[\rho^{2}+R^{2}\right]^{\frac{1}{2 b}-1} f \tilde{f}^{-\frac{1}{3}} \frac{\sqrt{1+R^{\prime 2}}}{\sqrt{\hat{B}^{2}+\left(\rho^{2}+R^{2}\right)^{\frac{2}{b}} \tilde{f}^{\frac{8}{3}}}},
$$

whereas for a black hole embedding we have:

$$
\mathcal{J}(m, \hat{B})=\frac{b r_{h}}{2^{\frac{2}{3}}} \int_{r_{h}}^{\infty} d r \sin ^{2} \theta \frac{\sqrt{1+h\left(\frac{r}{b}\right)^{2} \dot{\theta}^{2}}}{\sqrt{r^{4}+\left(\frac{r_{h}}{2^{\frac{2}{3}}}\right)^{4} \hat{B}^{2}}} .
$$

The internal energy density $E$ can be computed from the relation $E=F+T s$, with the result

$$
\frac{E}{\mathcal{N}}=2-2 \mathcal{G}(m, \hat{B})+2 \hat{B}^{2} \mathcal{J}(m, \hat{B})-(3-2 b) c m .
$$

The heat capacity density $c_{v}$ is defined as $c_{v}=\partial E / \partial T$. Computing explicitly the derivative of $E$ with respect to the temperature in (3.11), and using (3.6), we arrive at the following expression:

$$
\begin{aligned}
T \frac{c_{v}}{\mathcal{N}}= & 2 T \frac{s}{\mathcal{N}}-2 \hat{B}^{2}\left(\mathcal{J}(m, \hat{B})+2 \hat{B} \frac{\partial \mathcal{J}(m, \hat{B})}{\partial \hat{B}}\right) \\
& +(2 b-3)\left[\left(3-b-b \frac{\partial(\log c)}{\partial(\log m)}\right) c m-4 m \hat{B} \frac{\partial c}{\partial \hat{B}}\right]
\end{aligned}
$$


In order to holographically investigate the joint effect of the presence of flavors and magnetic field on the speed of sound, we use the following definition

$$
v_{s}^{2}=-\frac{\partial P}{\partial E}=\frac{\partial F}{\partial T}\left(\frac{\partial E}{\partial T}\right)^{-1}=\frac{s}{c_{v}} .
$$

Let us apply the formula (3.13) for the background plus probe system. Expanding at first order in the probe functions, we get:

$$
v_{s}^{2}=\frac{s_{\text {back }}+s}{c_{v, \text { back }}+c_{v}} \approx \frac{1}{2}-\frac{c_{v}-2 s}{4 s_{\text {back }}}
$$

where we have taken into account that $c_{v \text {,back }}=2 s_{\text {back }}$ and, therefore, $v_{s}^{2}=1 / 2$ for the background, as it corresponds to a conformal system in $2+1$ dimensions. Since, we can rewrite the ratio $\mathcal{N} / T s_{\text {back }}$ in the following form [19]

$$
\frac{\mathcal{N}}{T s_{\text {back }}}=\frac{1}{4} \frac{\lambda}{N} \frac{q}{b^{4}} \sigma^{2}
$$

we arrive at the following expression for the deviation $\delta v_{s}^{2}=v_{s}^{2}-\frac{1}{2}$ :

$$
\begin{aligned}
\delta v_{s}^{2} \approx \frac{\lambda}{N} \frac{q \sigma^{2}}{16 b^{4}}[ & 2 \hat{B}^{2}\left(\mathcal{J}(m, \hat{B})+2 \hat{B} \frac{\partial \mathcal{J}(m, \hat{B})}{\partial \hat{B}}\right) \\
& \left.+(3-2 b)\left(\left(3-b-b \frac{\partial(\log c)}{\partial(\log m)}\right) c m-4 m \hat{B} \frac{\partial c}{\partial \hat{B}}\right)\right],
\end{aligned}
$$

where we have used (3.12) to compute $c_{v}-2 s$ for the probe.

According to (3.4) the magnetization of the system is given by the following expression

$$
\mathcal{M}=-\left(\frac{\partial F}{\partial B}\right)_{T}=-\frac{2^{\frac{4}{3}} \pi}{3 b} \mathcal{N}_{r} T \hat{B} \mathcal{J}(m, \hat{B})
$$

The magnetic susceptibility $\chi$ is defined as:

$$
\chi \equiv \frac{\partial \mathcal{M}}{\partial B} .
$$

For generic embeddings, which are numerical, also the thermodynamic quantities need to be calculated numerically. However, there are two corners were analytic results can be obtained. The first one is when we study embeddings with asymptotically large $m$ and the other when the embeddings are massless. We will consider these two cases separately in the next two subsections.

\subsection{Massless embeddings}

For zero mass (and $c=0$ ) the embedding is necessarily a black hole embedding and it is more convenient to use the $\theta=\theta(r)$ parameterization. Actually, the massless embeddings 
in these variables are just characterized by the condition $\theta=\pi / 2$. Therefore, it follows from (3.3) that the function $\mathcal{G}(m=0, \hat{B})$ is given by the following integral:

$$
\mathcal{G}(m=0, \hat{B})=\frac{4 b}{r_{h}^{3}} \int_{r_{h}}^{\infty} d r r^{2}\left(\sqrt{1+\left(\frac{r_{h}}{2^{\frac{2}{3}} r}\right)^{4} \hat{B}^{2}}-1\right),
$$

which can be explicitly performed:

$$
\mathcal{G}(m=0, \hat{B})=\frac{4 b}{3}\left[1-{ }_{2} F_{1}\left(-\frac{1}{2},-\frac{3}{4}, \frac{1}{4} ;-\frac{\hat{B}^{2}}{2^{\frac{8}{3}}}\right)\right] .
$$

Then, it follows that the free energy is given by

$$
\frac{F}{\mathcal{N}}=-1+\frac{4 b}{3}\left[1-{ }_{2} F_{1}\left(-\frac{1}{2},-\frac{3}{4}, \frac{1}{4} ;-\left(\frac{3}{4 \pi}\right)^{4} \frac{B^{2}}{T^{4}}\right)\right] .
$$

Let us next compute the entropy density for the massless embeddings. By taking $m=0$ in $(3.8)$, we find

$$
T \frac{s(m=0, \hat{B})}{\mathcal{N}}=-3 \mathcal{G}(m=0, \hat{B})+2 \hat{B}^{2} \mathcal{J}(m=0, \hat{B})+3 .
$$

The integral $\mathcal{J}(m=0, \hat{B})$ can be evaluated explicitly from its definition (3.10),

$$
\mathcal{J}(m=0, \hat{B})=\frac{b}{2^{\frac{2}{3}}}{ }_{2} F_{1}\left(\frac{1}{4}, \frac{1}{2}, \frac{5}{4} ;-\frac{\hat{B}^{2}}{2^{\frac{8}{3}}}\right) .
$$

Plugging this result into (3.22), after some calculation, we arrive at the following simple expression for the entropy density of the massless embeddings:

$$
T \frac{s(m=0, B)}{\mathcal{N}}=3-4 b+4 b \sqrt{1+\left(\frac{3}{4 \pi}\right)^{4} \frac{B^{2}}{T^{4}}} .
$$

Similarly, the internal energy for zero mass is obtained from (3.11):

$$
\left.\frac{E}{\mathcal{N}}\right|_{m=0}=2+\frac{8 b}{3}\left[\sqrt{1+\left(\frac{3}{4 \pi}\right)^{4} \frac{B^{2}}{T^{4}}}-1+\left(\frac{3}{4 \pi}\right)^{4} \frac{B^{2}}{T^{4}}{ }_{2} F_{1}\left(\frac{1}{2}, \frac{1}{4}, \frac{5}{4} ;-\left(\frac{3}{4 \pi}\right)^{4} \frac{B^{2}}{T^{4}}\right)\right] .
$$

We will compute the heat capacity by taking $m=0$ in (3.12) and using the remarkable property:

$$
\mathcal{J}(m=0, \hat{B})+2 \hat{B} \frac{\partial \mathcal{J}(m=0, \hat{B})}{\partial \hat{B}}=\frac{b}{2^{\frac{2}{3}}} \frac{1}{\sqrt{1+\frac{\hat{B}^{2}}{2^{\frac{8}{3}}}}},
$$

which combined with (3.24) leads to the simple result:

$$
\left.T \frac{c_{v}}{\mathcal{N}}\right|_{m=0}=6+8 b\left[\frac{1}{\sqrt{1+\left(\frac{3}{4 \pi}\right)^{4} \frac{B^{2}}{T^{4}}}}-1\right] .
$$


This result can be confirmed by computing directly the derivative of the internal energy written in (3.25). The deviation of the speed of sound with respect to the conformal value $v_{s}^{2}=1 / 2$ is readily obtained from $(3.16)$ :

$$
\delta v_{s}^{2} \approx \frac{\lambda}{2 N} \frac{q \sigma^{2}}{b^{3}}\left(\frac{3}{4 \pi}\right)^{4} \frac{B^{2}}{T^{4} \sqrt{1+\left(\frac{3}{4 \pi}\right)^{4} \frac{B^{2}}{T^{4}}}}
$$

and the magnetization of the system at zero mass follows from (3.17) and (3.23):

$$
\mathcal{M}(m=0, B)=-\frac{3 \mathcal{N}_{r}}{4 \pi} \frac{B}{T}{ }_{2} F_{1}\left(\frac{1}{4}, \frac{1}{2}, \frac{5}{4} ;-\left(\frac{3}{4 \pi}\right)^{4} \frac{B^{2}}{T^{4}}\right) .
$$

We note that the magnetization is always negative and vanishes at zero field. It is no surprise that the system is diamagnetic. The magnetic field appears with even power inside the DBI action, which implies that the spontaneous magnetization vanishes. Moreover, the DBI action has a specific (plus) sign, meaning that the magnetization is always nonpositive. $^{2}$

To obtain the magnetic susceptibility we have to compute the derivative of the righthand side of (3.29) with respect to the magnetic field (see (3.18)). We get:

$$
\chi(m=0, B)=-\frac{3 \mathcal{N}_{r}}{8 \pi T}\left[\frac{1}{\sqrt{1+\left(\frac{3}{4 \pi}\right)^{4} \frac{B^{2}}{T^{4}}}}+{ }_{2} F_{1}\left(\frac{1}{4}, \frac{1}{2}, \frac{5}{4} ;-\left(\frac{3}{4 \pi}\right)^{4} \frac{B^{2}}{T^{4}}\right)\right] .
$$

In the equations written above for the massless black hole embeddings the dependence on the number of flavors is contained implicitly in the parameter $b$ (see (2.6)), while the dependence on the magnetic field and temperature is manifest. One can take further limits in some of these functions. For example, the $T=0$ values of the free energy (3.21) and entropy (3.22) are:

$$
\left.\frac{F(m=0)}{\mathcal{N}_{r}}\right|_{T=0}=\frac{B^{3 / 2}}{6 \sqrt{\pi}} \Gamma\left[\frac{1}{4}\right]^{2},\left.\quad s(m=0)\right|_{T=0}=\frac{4 \pi}{3} \mathcal{N}_{r} B,
$$

while the magnetization (3.29) of the massless embeddings at zero temperature is:

$$
\left.\mathcal{M}(m=0)\right|_{T=0}=-\frac{1}{4 \sqrt{\pi}} \mathcal{N}_{r} \Gamma\left[\frac{1}{4}\right]^{2} \sqrt{B}
$$

Let us pause here for a while. We wish to emphasize, that though we were able to produce analytic formulas in the special case of massless embedding, this phase is only relevant for small values of $\hat{B}$. In particular, the $T=0$ case is never thermodynamically preferred. The phase diagram will be addressed in section 4 .

\footnotetext{
${ }^{2}$ In other systems, where the gauge fields have Chern-Simons terms, their contribution to the magnetization can be positive thus leading to a competition with the DBI part. As a result one might get a positive overall magnetization leading to paramagnetism (see [24, 25]), or even to ferromagnetism (as in [25]).
} 


\subsubsection{Small magnetic field}

Let us focus on limits of thermodynamic quantities for the massless case when the magnetic field is small (actually when $B / T^{2} \rightarrow 0$ ). These expressions give the first correction, due to the magnetic field, to the conformal behavior of the probe at $B / T^{2} \rightarrow 0$. For $F, s, E$, and $c_{v}$ we find

$$
\begin{array}{ll}
\frac{F}{\mathcal{N}} \approx-1+2 b\left(\frac{3}{4 \pi}\right)^{4} \frac{B^{2}}{T^{4}}, & T \frac{s}{\mathcal{N}} \approx 3+2 b\left(\frac{3}{4 \pi}\right)^{4} \frac{B^{2}}{T^{4}}, \\
\frac{E}{\mathcal{N}} \approx 2+4 b\left(\frac{3}{4 \pi}\right)^{4} \frac{B^{2}}{T^{4}}, & T \frac{c_{v}}{\mathcal{N}} \approx 6-4 b\left(\frac{3}{4 \pi}\right)^{4} \frac{B^{2}}{T^{4}} .
\end{array}
$$

Moreover, the variation of the speed of sound at leading order in $B / T^{2}$ is:

$$
\delta v_{s}^{2} \approx \frac{\lambda}{2 N} \frac{q \sigma^{2}}{b^{3}}\left(\frac{3}{4 \pi}\right)^{4} \frac{B^{2}}{T^{4}}, \quad\left(B / T^{2} \rightarrow 0\right)
$$

and the magnetization becomes:

$$
\mathcal{M} \approx-\frac{3 \mathcal{N}_{r}}{4 \pi} \frac{B}{T}=-\frac{3(2-b) b^{2} \sigma}{(4 \pi)^{2} \sqrt{2}} N \sqrt{\lambda} \frac{B}{T}, \quad\left(B / T^{2} \rightarrow 0\right)
$$

It follows that the susceptibility at vanishing magnetic field is:

$$
\chi(m=B=0)=-\frac{3(2-b) b^{2} \sigma}{(4 \pi)^{2} \sqrt{2}} N \sqrt{\lambda} \frac{1}{T} .
$$

Thus, the diamagnetic response of the system goes to zero as the temperature approaches infinity. The behavior possessed by (3.36) closely resembles another $(2+1)$-dimensional construction [27]. In both cases the system behaves as in Curie's law $\chi \propto 1 / T$, though they are diamagnetic. From a dimensional analysis point of view this temperature dependence is the expected one for the magnetic susceptibility in $2+1$ dimensions, since at high $T$ conformality is restored.

\subsection{Approximate expressions for large mass}

When the D6-brane probe remains far away from the horizon, it is possible to obtain analytic results for the free energy and the rest of the thermodynamic quantities. Following the analysis of $[19,26]$, for large $m$ the embeddings are nearly flat and given by the following expression

$$
R(\rho)=R_{0}+\delta R(\rho),
$$

where $R_{0}$ is a constant and $\delta R(\rho)$ is much smaller than $R_{0}$. Before calculating the free energy we want an approximate expression for the condensate of the theory as a function of the mass and the magnetic field. For this task we need the relationship between $R_{0}$ and $m$. A simple calculation yields the following expansion in powers of $m$

$$
R_{0}=m-a(b) m^{1-\frac{6}{b}}+\frac{1}{4} a_{1}(b) \hat{B}^{2} m^{1-\frac{4}{b}}+\cdots,
$$


where the function $a(b)$ is given in equation (B.15) of [19], which we record here for completeness and $a_{1}(b)$ is

$$
\begin{aligned}
a(b) & =\frac{3}{3+2 b}\left[\frac{2 b}{3-2 b}+\psi\left(\frac{3}{b}\right)-\psi\left(\frac{3}{2 b}\right)\right] \\
a_{1}(b) & =\frac{2 b}{3-2 b}-\psi\left(\frac{3}{2 b}\right)+\psi\left(\frac{2}{b}\right),
\end{aligned}
$$

where $\psi(x)=\Gamma^{\prime}(x) / \Gamma(x)$ is the digamma function. Using (3.38) it is possible to obtain an approximate expression for the condensate as a function of the mass for any number of flavors

$$
c=\frac{6 b}{4 b^{2}-9} m^{-1-\frac{3}{b}}+\frac{1}{2} \frac{b \hat{B}^{2}}{3-2 b} m^{-1-\frac{1}{b}}-\frac{4}{3} \frac{b \hat{B}^{2}}{3-2 b} m^{-1-\frac{4}{b}}+\cdots .
$$

Using these results in (3.2) and (3.9) it is possible to evaluate the functions $\mathcal{G}(m, \hat{B})$ and $\mathcal{J}(m, \hat{B})$ for large values of the mass parameter $m$. For $\mathcal{G}$ we get

$$
\mathcal{G}(m, \hat{B})=1+\frac{b}{2} \frac{\hat{B}^{2}}{m^{\frac{1}{b}}}-\frac{2 b}{3+2 b} \frac{1}{m^{\frac{3}{b}}}-\frac{b}{3} \frac{\hat{B}^{2}}{m^{\frac{4}{b}}}+\cdots,
$$

while $\mathcal{J}$ behaves for large $m$ as:

$$
\mathcal{J}(m, \hat{B})=b\left[\frac{1}{m^{\frac{1}{b}}}-\frac{2}{3} \frac{1}{m^{\frac{4}{b}}}\right]+\cdots .
$$

It is now straightforward to compute the different thermodynamic functions in this high mass regime. Indeed, the free energy and the entropy follow directly by substituting (3.42) and (3.43) into (3.1) and (3.8), respectively,

$$
\begin{aligned}
\frac{F}{\mathcal{N}} & =-\frac{2 b}{3+2 b} \frac{1}{m^{\frac{3}{b}}}+\frac{b}{2} \frac{\hat{B}^{2}}{m^{\frac{1}{b}}}-\frac{b}{3} \frac{\hat{B}^{2}}{m^{\frac{4}{b}}} \cdots \\
T \frac{s}{\mathcal{N}} & =\frac{12 b}{3+2 b} \frac{1}{m^{\frac{3}{b}}}+\frac{b \hat{B}^{2}}{m^{\frac{4}{b}}}+\cdots .
\end{aligned}
$$

Similarly, $E$ and $c_{v}$ can be expanded as

$$
\begin{aligned}
\frac{E}{\mathcal{N}} & =\frac{b}{2} \frac{\hat{B}^{2}}{m^{\frac{1}{b}}}+\frac{10 b}{3+2 b} \frac{1}{m^{\frac{3}{b}}}+\frac{2 b}{3} \frac{\hat{B}^{2}}{m^{\frac{4}{b}}}+\cdots \\
T \frac{c_{v}}{\mathcal{N}} & =\frac{60 b}{3+2 b} \frac{1}{m^{\frac{3}{b}}}+2 b \frac{\hat{B}^{2}}{m^{\frac{4}{b}}}+\cdots,
\end{aligned}
$$

and the variation of the speed of sound at large $m$ is given by

$$
\delta v_{s}^{2}=-\frac{9}{4} \frac{\lambda}{N} \frac{\sigma^{2}}{(3+2 b)(2-b) b^{2}} \frac{1}{m^{\frac{3}{b}}}+\cdots .
$$

Finally, in the high mass regime the magnetization can be expanded as:

$$
\mathcal{M}=-\frac{2^{\frac{4}{3}} \pi}{9} \mathcal{N}_{r} T \hat{B}\left(\frac{3}{m^{\frac{1}{b}}}-\frac{2}{m^{\frac{4}{b}}}\right) \cdots .
$$

This regime of large $m$ is achieved when the temperature is low. Therefore, it is interesting to compare these results with the ones obtained when $T=0$. We will perform this analysis in the next subsection. 


\subsubsection{Zero temperature limit}

One can calculate the different thermodynamic functions at zero temperature by working directly with the parameterization of section 2.4, in which the embeddings are characterized by the two rescaled parameters $\tilde{m}_{0}$ and $\tilde{c}_{0}$ (see (2.36) and (2.37)). For large values of $\tilde{m}_{0}$ one can proceed as above and find an approximate expression of the condensate as a function of the mass:

$$
\tilde{c}_{0}=\frac{1}{2} \frac{b}{3-2 b} \tilde{m}_{0}^{-1-\frac{1}{b}}+\cdots, \quad\left(\tilde{m}_{0} \rightarrow \infty\right) .
$$

Notice that, according to our dictionary (2.46), large $\tilde{m}_{0}$ corresponds to large $\mu_{q}^{0}$ or small $B$. Actually, we can extract the dependence of the condensate on the $B$ field by rewriting (3.50) in terms of the unrescaled parameters $c_{0}$ and $m_{0}$

$$
c_{0}=\frac{1}{2} \frac{b}{3-2 b} B^{2} m_{0}^{-1-\frac{1}{b}}+\cdots .
$$

It is worth pointing out that (3.50) and (3.51) can also be obtained by using (2.52) and keeping the leading terms in the $T \rightarrow 0$ limit. It is instructive to write (3.51) in terms of physical quantities. We can use our dictionary (2.46) and (2.47) to translate (3.51) to

$$
-\frac{\left\langle\mathcal{O}_{q}\right\rangle_{0}}{N}=\frac{(2-b) b}{8 \pi^{2}} \frac{B_{\text {phys }}}{\sqrt{2 \lambda}}\left[\frac{\sqrt{\lambda}}{2 \sqrt{2} \pi \sigma} \frac{B_{\text {phys }}}{\left(\mu_{q}^{0}\right)^{2}}\right]^{\frac{b+1}{2 b}},
$$

where we have written the result in terms of $B_{\text {phys }}=L^{2} B$. Similarly, by direct calculation or by using the limiting expressions $(2.52)$, one finds that the free energy for large $\tilde{m}_{0}$ can be approximated as:

$$
\frac{F}{\mathcal{N}_{r}}=\frac{1}{2} B^{2} m_{0}^{-\frac{1}{b}}+\cdots,
$$

which, in terms of physical quantities corresponds to

$$
F=\frac{(2-b) b^{2}}{8 \pi^{2} \sqrt{2}} \frac{N}{\sqrt{\lambda}} \mu_{q}^{0} B_{\text {phys }}\left[\frac{\sqrt{\lambda}}{2 \sqrt{2} \pi \sigma} \frac{B_{\text {phys }}}{\left(\mu_{q}^{0}\right)^{2}}\right]^{\frac{b+1}{2 b}}+\cdots .
$$

By computing the derivatives with respect to the magnetic field of the free energy written above one can easily obtain the magnetization and susceptibility at zero temperature in the regime in which $\mu_{q}^{0} / \sqrt{B}$ is large.

\section{Magnetic catalysis}

In this section we will address the full phase diagram of the system at non-zero magnetic field, zero and non-zero temperature, and in the presence of background smeared flavors $N_{f} \neq 0$. There are excellent reviews $[10,11]$ which discuss some of the interesting phenomena that occur on probe-brane systems when an external magnetic field is turned on. To narrow the scope we focus on a particular effect where the magnetic field induces spontaneous chiral symmetry breaking, known as the magnetic catalysis [5-7]. Only rather recently have we witnessed attempts in addressing magnetic catalysis that arise away from 
the probe limit $[13,14]$. Current paper constitutes our second step in this direction, the first one being the construction of a dual to unquenched massive flavors [20]. Ultimately, one wishes to combine the two and also backreact the magnetic field on the supergravity solution. Our goal is very ambitious, but we nevertheless feel that it should not go without serious attempt. In the current paper, we are more modest and consider the magnetic field only residing on the probe, but as a background we consider the fully backreacted massless flavored ABJM model.

We will begin our discussion with zero temperature case and then move on to non-zero temperature. Our findings resemble somewhat the results in the supersymmetric D3-D7 probe brane analysis $[10,11]$. However, surprisingly, the flavor factors go along the ride and we can thus analyze the background flavor effects exactly, in contrast to D3-D7 system where the flavors have to be treated perturbatively $[13,14]$. We also show that the magnetic catalysis is enhanced (suppressed) with flavor effects at large (small) magnetic field strength when the bare quark mass is non-zero.

\subsection{Zero temperature}

Consider the equation of motion for the embedding $\tilde{R}=\tilde{R}(\tilde{\rho})$ as derived from the action (2.34) at zero temperature $T=0$. At non-zero $B$ the supersymmetry is broken, and thus the D6-brane has a profile (as in (2.35)) which depends on $\tilde{\rho}$. For large $\tilde{m}_{0}$, the condensate can be obtained analytically (see (3.50)), but for small values of $\tilde{m}_{0}$, the $\tilde{c}_{0}$ needs to be numerically solved for. In figure 1 (left panel) we display the parametric plot of $\tilde{c}_{0}$ versus $\tilde{m}_{0}$, which was generated by varying the IR value $\tilde{R}(0)=\tilde{R}_{0}$ and shooting towards the AdS boundary. ${ }^{3}$ In figure 1 (right panel) we plot the free energy as a function of $\tilde{m}_{0}$. We find several possible solutions for some given small $\tilde{m}_{0}$, but immediately infer that the physical solution is the one with lowest free energy. This corresponds to the solution with larger condensate, i.e., corresponding to the right arms of $\left(\tilde{m}_{0}, \tilde{c}_{0}\right)$ curves. We also note, that the large $\tilde{m}_{0}$ tail corresponds to the analytic behavior (3.50), whereas zooming in toward origin of the plot would probably result in a self-similar behavior of the equation of state, similarly as was analyzed in [19].

\subsubsection{Zero bare mass}

An important result is that the $\tilde{m}_{0}=0$ embedding has a non-zero fermion condensate $\tilde{c}_{0} \propto\left\langle\mathcal{O}_{q}\right\rangle_{0}$. In terms of physical quantities, the relation (2.47) between $\left\langle\mathcal{O}_{q}\right\rangle_{0}$ and $\tilde{c}_{0}$ has been written in the appendix (eq. (A.7)). The introduction of the magnetic field $B$ has therefore induced a spontaneous chiral symmetry breaking.

We now focus on the flavor effects. While we find in figure 1 that the $\tilde{c}_{0}$ grows with increasing $\hat{\epsilon}$, the condensate $\left\langle\mathcal{O}_{q}\right\rangle_{0}$ given in (A.7) actually has the opposite behavior with more flavor. In figure 2 we plot essentially $\left\langle\mathcal{O}_{q}\right\rangle_{0}$ against $\hat{\epsilon}$ and see that the condensate actually decreases monotonously with $\hat{\epsilon}$ for fixed $B_{\text {phys }}$. In a different system [13] the tendency for the condensate to decrease with flavor was also observed. At infinite flavor $\hat{\epsilon} \rightarrow \infty,\left\langle\mathcal{O}_{q}\right\rangle_{0}$ reaches a constant non-zero value.

\footnotetext{
${ }^{3}$ Notice that only $\tilde{m}_{0} \geq 0$ embeddings are physical; otherwise the angle $\theta>\pi / 2$.
} 

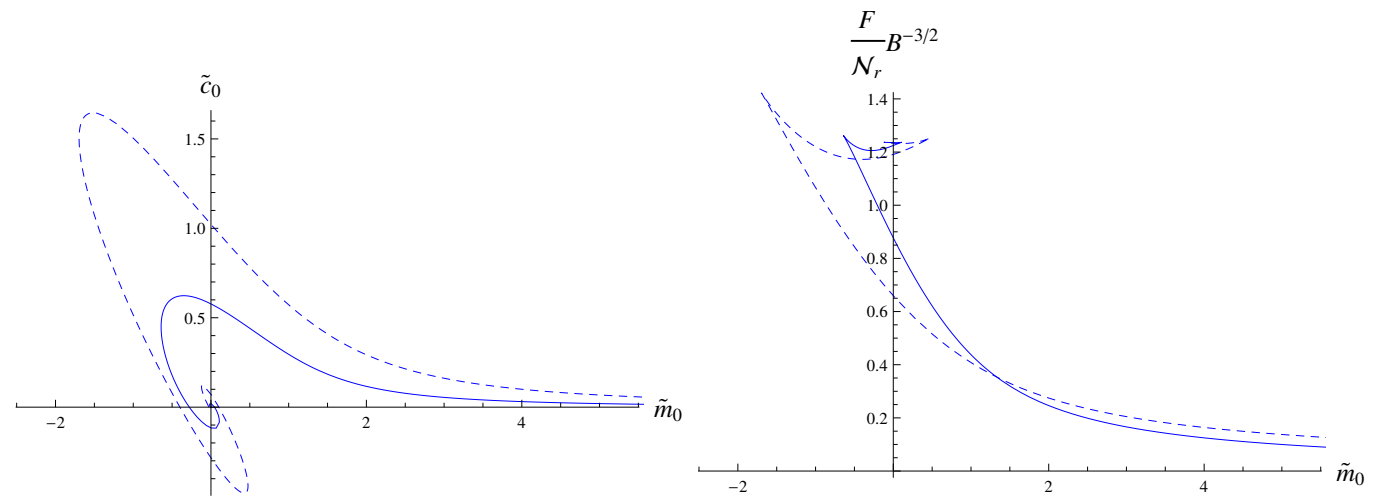

Figure 1. Plot of the condensate $\tilde{c}_{0}$ (left) and the free energy (right) versus the rescaled mass $\tilde{m}_{0}$. The solid blue is $\hat{\epsilon}=0$ and the dashed blue is $\hat{\epsilon}=\infty$. Both of the curves on the right panel start at the value (at $\left.\tilde{m}_{0}=0\right) \frac{\Gamma(1 / 4)^{2}}{6 \sqrt{\pi}}$ as extracted from (3.31). Notice that there is no phase transition, since for all $\tilde{m}_{0} \geq 0$ we reside on the same solution.

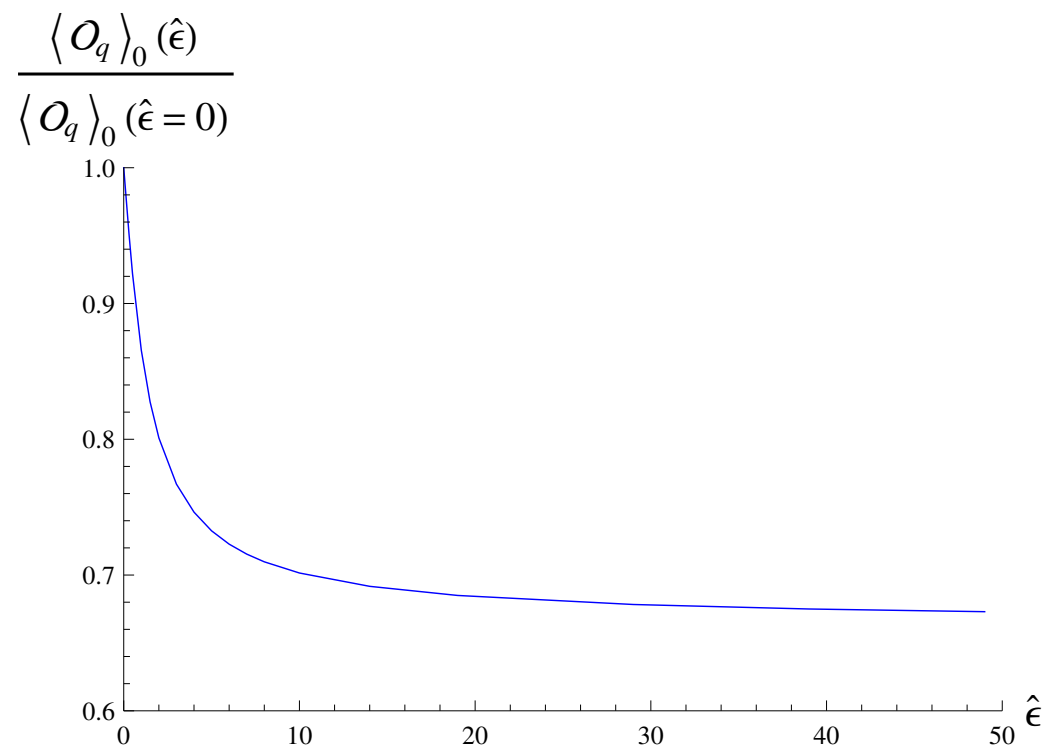

Figure 2. Plot of the condensate against the deformation parameter at zero bare mass. At infinite flavor the condensate reaches a constant value for fixed physical magnetic field $B_{\text {phys }}$. Notice that we normalized the depicted quantity to unity in the quenched limit.

\subsubsection{Non-zero bare mass}

While the magnetic catalysis is the main focus of this paper, it is interesting to study the case with non-vanishing bare mass. In other words, we wish to study the system when the chiral symmetry is explicitly broken, rather than spontaneously, and ask what does the condensate care about the magnetic field and background flavor.

When the bare mass is non-zero it is convenient to study the value of the condensate for a fixed value of $\mu_{q}^{0}$. From our dictionary $(2.46)$ we can relate the mass parameter $\tilde{m}_{0}$ 

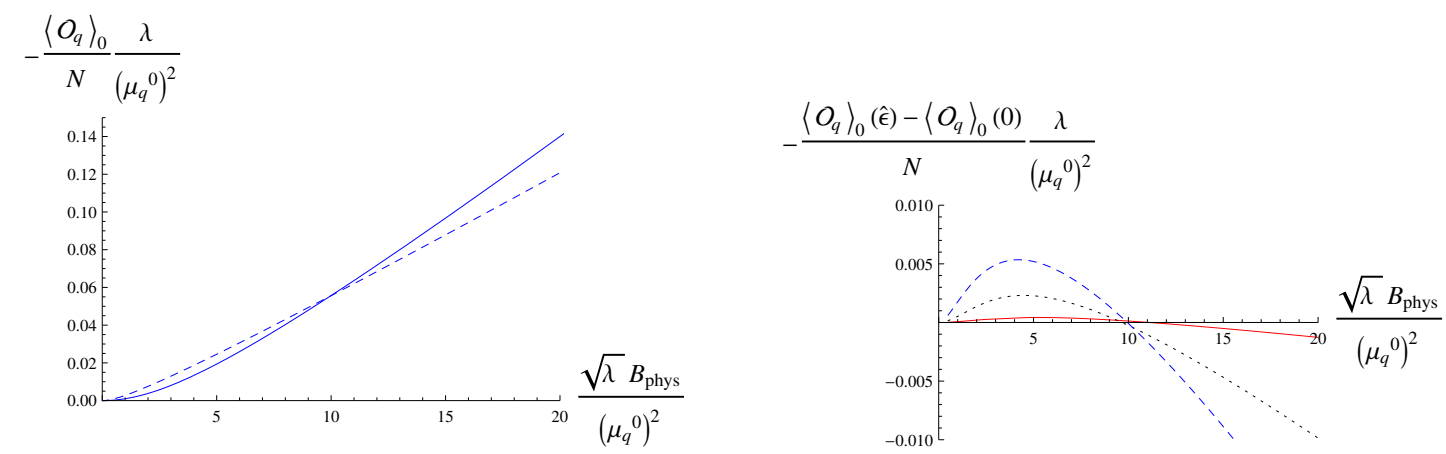

Figure 3. Plots of the condensate versus the magnetic field, when the bare quark mass is non-zero. The solid blue is $\hat{\epsilon}=0$ and the dashed blue is $\hat{\epsilon}=10$. In the right panel we have also included the $\hat{\epsilon}=0.1$ (solid red) and the $\hat{\epsilon}=1$ (dotted black) curves.

to $\mu_{q}^{0}$ and the physical magnetic field. We get:

$$
\tilde{m}_{0}=\sqrt{2 \sqrt{2} \pi \sigma}\left[\frac{\left(\mu_{q}^{0}\right)^{2}}{\sqrt{\lambda} B_{\mathrm{phys}}}\right]^{\frac{1}{2}}
$$

The formula (4.1) enables us to plot the condensate against the magnetic field itself, in units of $\mu_{q}^{0}$ rather than the rescaled mass $\tilde{m}_{0}$. Indeed, let us now consider the quantity:

$$
-\frac{\lambda}{\left(\mu_{q}^{0}\right)^{2}} \frac{\left\langle\mathcal{O}_{q}\right\rangle_{0}}{N}=\frac{(3-2 b)(2-b)}{4 \pi^{2} \sqrt{2}} \frac{\sqrt{\lambda} B_{\text {phys }}}{\left(\mu_{q}^{0}\right)^{2}} \tilde{c}_{0} .
$$

The condensate parameter $\tilde{c}_{0}$ depends non-trivially on $\tilde{m}_{0}$ (the precise dependence must be found by numerical calculations), which in turn can be written as in (4.1). Thus, it follows that the left-hand side of (4.2) depends on $\sqrt{\lambda} B_{\text {phys }} /\left(\mu_{q}^{0}\right)^{2}$.

In figure 3 we show the condensate against the magnetic field and find that it increases monotonously. Moreover, for small $B_{\text {phys }} /\left(\mu_{q}^{0}\right)^{2}$ the condensate for the flavored theory is larger than the unflavored one. Thus, for small $B_{\text {phys }}$ or large $\mu_{q}^{0}$, the flavors produce an enhancement of the chiral symmetry breaking. In figure 3 we illustrate this flavor effect by plotting the difference of condensates as a function of $B_{\text {phys }} /\left(\mu_{q}^{0}\right)^{2}$. Actually, for small values of $B_{\text {phys }} /\left(\mu_{q}^{0}\right)^{2}$ we can use the approximate expression (3.50) to estimate $\tilde{c}_{0}$. Plugging (3.50) and (4.1) into (4.2) we get for small $B_{\text {phys }} /\left(\mu_{q}^{0}\right)^{2}$

$$
-\frac{\lambda}{\left(\mu_{q}^{0}\right)^{2}} \frac{\left\langle\mathcal{O}_{q}\right\rangle_{0}}{N} \approx \frac{(2-b) b}{8 \sqrt{2} \pi^{2}}(2 \pi \sqrt{2} \sigma)^{-\frac{1}{2}-\frac{1}{2 b}}\left[\frac{\sqrt{\lambda} B_{\text {phys }}}{\left(\mu_{q}^{0}\right)^{2}}\right]^{\frac{3}{2}+\frac{1}{2 b}} .
$$

Thus, we get a power law behavior with an exponent which depends on the number of flavors and matches the numerical results.

For large values of $B_{\text {phys }} /\left(\mu_{q}^{0}\right)^{2}$ the flavors suppress the condensate and we have a behavior similar to the massless case. Curiously, this change of behavior occurs at values of $B_{\text {phys }} /\left(\mu_{q}^{0}\right)^{2}$ which are almost independent of the number of flavors (see figure 3 ). 


\subsection{Non-zero temperature}

Having understood the basic physics behind introducing the magnetic field, let us now heat up the system and study what happens. In addition to the MN embeddings, we now also have the $\mathrm{BH}$ embeddings at our disposal. At zero magnetic field, $B=0$, we recall that there is going to be a phase transition from the $\mathrm{MN}$ phase to the $\mathrm{BH}$ phase as the temperature is increased [19]. The black hole begins to increasingly attract the probe D6brane. Turning on $B$ has the opposite effect, in some sense the magnetic field makes the D6-brane to repel. We thus have two competing effects in play and we need to explore the four-dimensional phase space $\left(T, B, \mu_{q}, \hat{\epsilon}\right)$, to find out which phase is thermodynamically preferred. Recall that at non-zero temperature we can form the dimensionless ratio (2.26), which for the physical magnetic field is $\hat{B}_{\text {phys }} \equiv 2^{4 / 3} \frac{B_{\text {phys }}}{r_{h}^{2}}$ and that the bare mass $\mu_{q}$ was introduced in [19], see below. This narrows down the phase space down to three dimensions $\left(\hat{B}_{\text {phys }}, \mu_{q}, \hat{\epsilon}\right)$. Let us begin our journey in the simpler case with vanishing bare quark mass $\mu_{q}=0$.

\subsubsection{Zero bare mass}

We start exploring the phase space in the case where we set $\mu_{q}=0$. This slice of the full phase diagram is easily obtained. At any given $\hat{\epsilon}$ we only have two options, either the system is in the chirally symmetric $\mathrm{BH}$ phase (small $\hat{B}_{\text {phys }}$ ) or the system is in the MN phase (large $\hat{B}_{\text {phys }}$ ) and the chiral symmetry is broken; see figure 4 . There is a first order phase transition at some critical $\hat{B}_{\text {crit }}^{\text {phys }}$, which depends on $\hat{\epsilon}$. Above this critical $\hat{B}_{\text {crit }}^{\text {phys }}$, the $\mathrm{BH}$ phase is never reached and thus the chiral symmetry is spontaneously broken. The phase diagram $(\hat{\epsilon}, \hat{B})$ is presented in figure 5 (left panel). The curve plotted $\hat{B}_{\text {crit }}^{\text {phys }}=\hat{B}_{\text {crit }}^{\text {phys }}(\hat{\epsilon})$ shows that the critical magnetic field decreases with increasing number of flavors. In other words, at fixed temperature, the more flavor there is the smaller magnetic field is needed to realize magnetic catalysis. As a consequence the critical condensate will also be smaller with more flavors, as is visible in figure 5 (right panel).

We finish this subsection by presenting the graph figure 6 , which represents the condensate as a function of the magnetic field at selected flavor deformation parameters $(\hat{\epsilon}=0$ and 10) and zero bare quark mass. The swallow-tail structures of the free energy graphs are indications of the first order phase transition, and from figure 6 we conclude that the condensate acts as an order parameter: at critical $\hat{B}_{\text {crit }}^{\text {phys }}$ the condensate jumps to a non-zero value and increases thereafter. From the numerics we also infer, that for large $\hat{B}$,

$$
\frac{\left\langle\mathcal{O}_{q}\right\rangle}{N} \sim T^{2} \hat{B}^{(3-b) / 2} \sim B \cdot\left(\frac{T}{\sqrt{B}}\right)^{\gamma_{m}} \quad, \hat{B} \gg 1 .
$$

This behavior conforms with the $T=0$ result $(2.47)$ at $m=0$ (recall the relation (2.56)).

\subsubsection{Non-zero bare mass}

To complete the investigation of the phase diagram, let us turn on a non-zero bare mass at non-zero temperature. Recall that the bare mass $\mu_{q}$ is given by [19]

$$
\frac{\mu_{q}}{T \sqrt{\lambda}}=\frac{2^{5 / 6} \pi}{3} m
$$



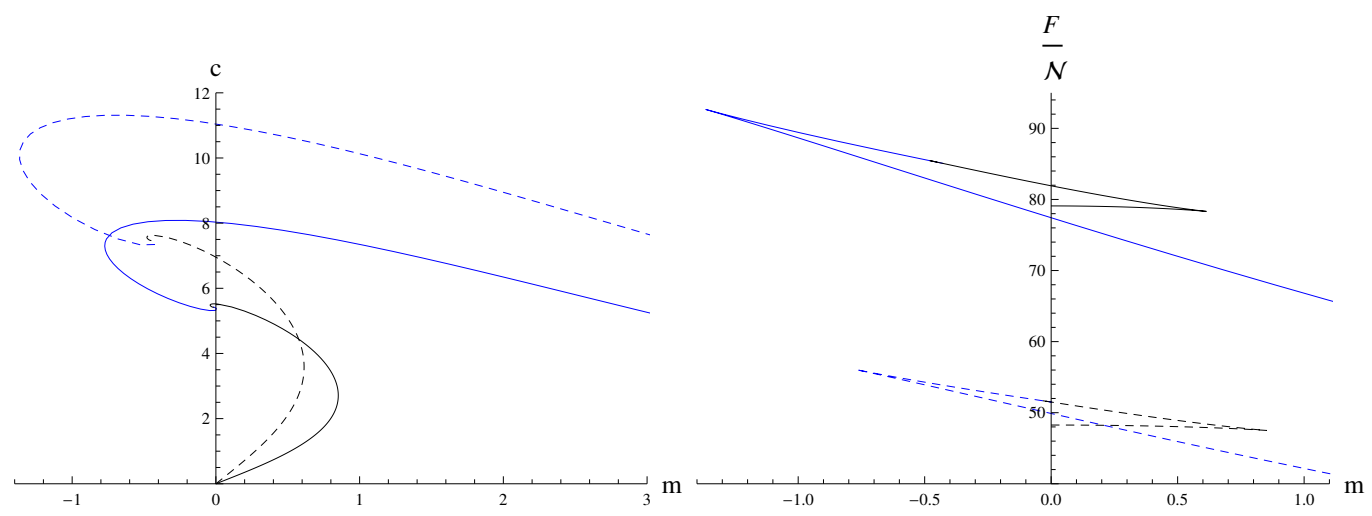

Figure 4. Plot of the condensate $c$ (left) and the free energy (right) versus $m$ at $\hat{\epsilon}=0$. The solid curves are for $\hat{B}=20$ and the dashed curves are for $\hat{B}=15$. The blue color stands for MN embeddings and black for BH embeddings. The phase transition for $m=0$ is between these two cases, around $\hat{B}_{\text {crit }}(\hat{\epsilon}=0) \sim 17.8$, above this critical value the chiral symmetry is always broken.
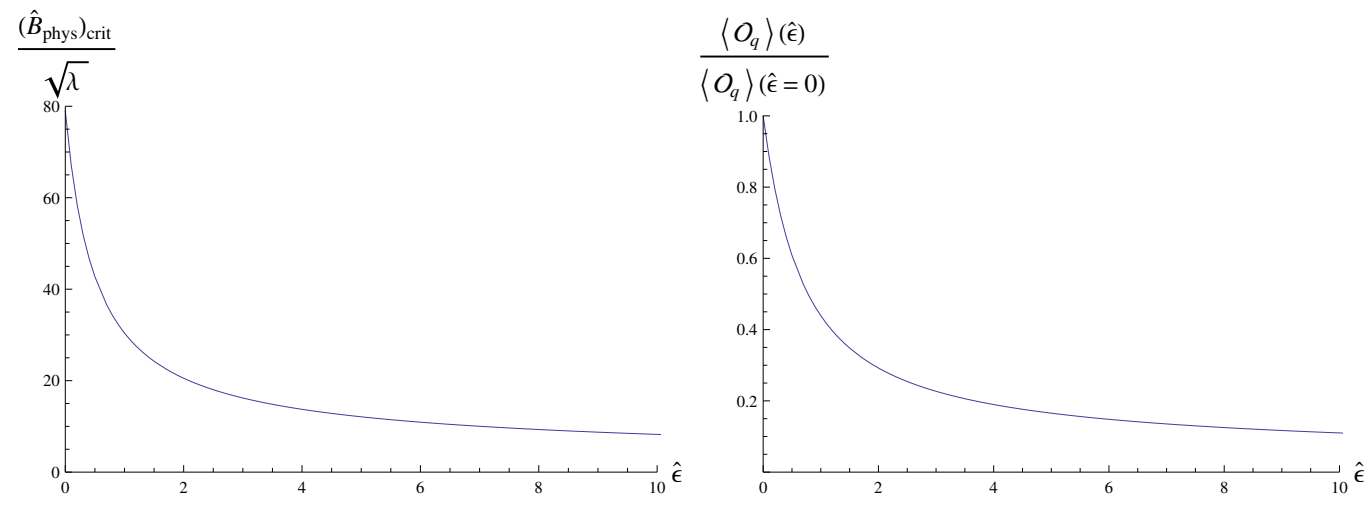

Figure 5. On the left we plot the phase diagram for $m=0$ in the $(\hat{\epsilon}, \hat{B})$-plane. Above the curve, the chiral symmetry is spontaneously broken whereas below the curve the condensate is zero. We note that the critical magnetic field needed, at fixed temperature, to break the chiral symmetry decreases with the number of flavors, leading to the decrease of the condensate (and asymptotically vanishing due to the screening function $\sigma$ ), as depicted on the right.

Given the relation (4.5), instead of directly fixing the bare mass to some value, we can fix $m$ (for any flavor deformation parameter $\hat{\epsilon}$ ). We just need to keep in mind that larger $m$ will then correspond to smaller temperatures, and vice versa.

We anticipate that there are essentially two different cases, depending on whether $m$ is small or large. In figure 7 we depict the condensate as a function of $\hat{B}$ for various $m$ at $\hat{\epsilon}=0$; the $\hat{\epsilon}>0$ is qualitatively the same with smaller $\hat{B}_{\text {crit }}$ 's. We find that for any given $\hat{\epsilon}$ there exists a large enough $m$ such that the system is always in the chirally broken MN phase for any $\hat{B}$. For small values of $m$, there can be a phase transition from the chirally symmetric $\mathrm{BH}$ phase to a broken $\mathrm{MN}$ phase at some critical $\hat{B}_{\text {crit }}$.

\section{Conclusions}

Let us shortly recap the main results of our work. We studied the ABJM Chern-Simons matter model with dynamical flavors, added as smeared flavor D6-branes. Our black hole 


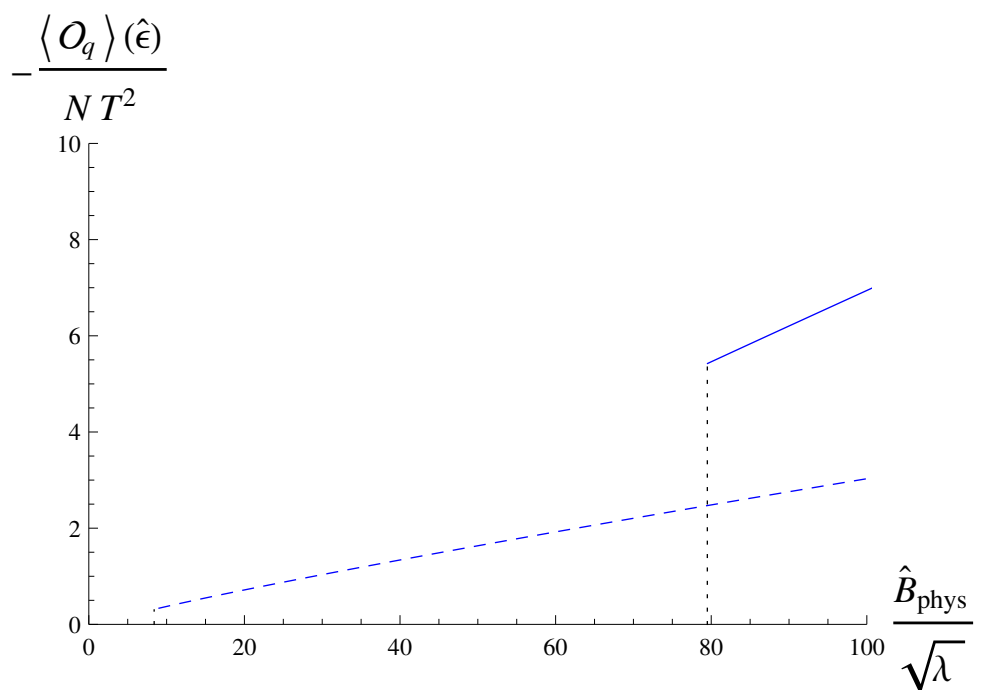

Figure 6. The condensate versus the magnetic field $\hat{B}$ at $\mu_{q}=0$. The solid blue curve is for $\hat{\epsilon}=0$ and the dashed blue curve is for $\hat{\epsilon}=10$. On the left of the curves the condensate is zero. Notice that at critical magnetic field $\hat{B}_{\text {crit }}(\hat{\epsilon})$ there is a first order phase transition (from the chirally symmetric $\mathrm{BH}$ phase) where the condensate jumps to a non-zero value, thus acting as an order parameter for the transition (to the chirally broken MN phase).

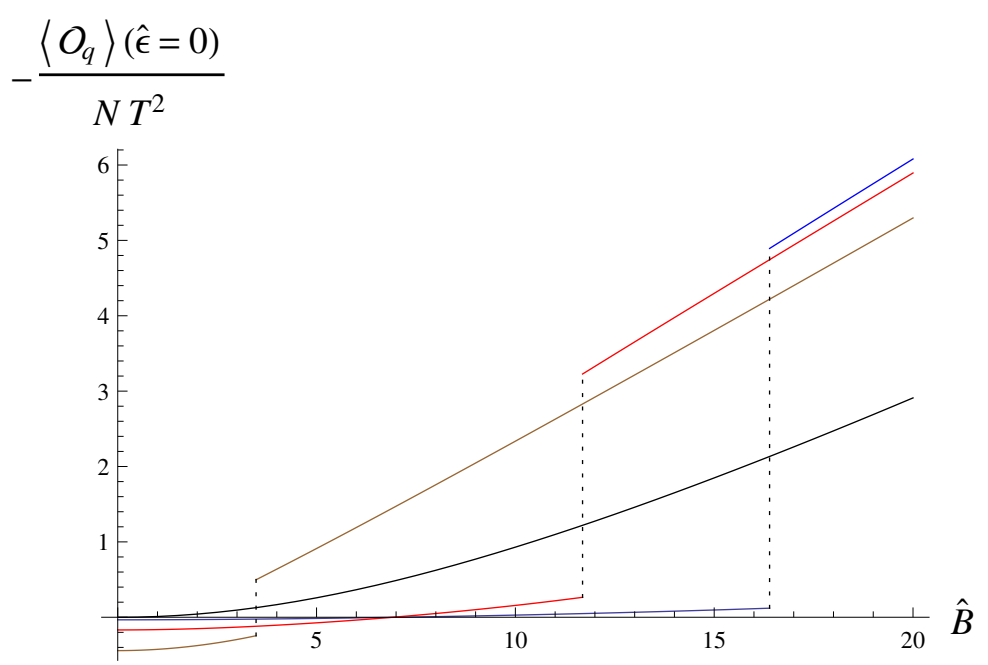

Figure 7. The condensate versus the magnetic field $\hat{B}$ at various fixed bare masses at quenched case $\hat{\epsilon}=0$. The critical magnetic field $\hat{B}_{\text {crit }}$, whose values correspond to the dotted vertical line segments, decreases as $m$ increases, and the respective curves read $m=0.1$ (blue), $m=0.5$ (red), $m=1$ (brown), and $m=5$ (continuous black).

geometry includes the backreaction of dynamical massless flavors at fully non-linear order in the flavor deformation parameter (2.7). We investigated the effect of the inclusion of an external magnetic field on the worldvolume of an (additional) probe D6-brane and focused on the flavor effects from the smeared D6-branes of the background. We obtained the different thermodynamic functions for the probe and explored the corresponding phase diagram. In some corners of this phase space we were able to obtain analytic results. 
At zero temperature, for any magnetic field strength, the system was always in the chirally broken MN phase; a phenomenon called magnetic catalysis. At large (small) magnetic field strength, at non-vanishing bare quark mass, the condensate was found decreasing (increasing) with the number of flavors. In other words, for small masses the magnetic catalysis is suppressed whereas for large values of the mass it is enhanced given more flavors in the background. This behavior could morally be thought of as inverse magnetic catalysis in the sense of $[28,29]$, although is technically different.

At non-zero temperature there was a critical magnetic field above which the magnetic catalysis took place. The condensate acted as an order parameter for the first order phase transition between the transition from the chirally symmetric $\mathrm{BH}$ phase to the broken MN phase. We found that the critical magnetic field was smaller for more flavors, which we interpret as an enhancement of the magnetic catalysis.

Let us finally discuss some possible extensions of our work. First of all, we could analyze the effects of having unquenched massive flavors. The corresponding background for the ABJM theory at zero temperature has been recently constructed in [20]. It would be interesting to explore in this setup how the flavor effects on the condensate are enhanced or suppressed as the mass of the unquenched quarks is varied, and to compare with the results found here in section 4.1.2. Also, one could try to include the effect of the magnetic field on the unquenched quarks. For this purpose a new non-supersymmetric background must be constructed first (see $[13,14]$ for a similar analysis in the D3-D7 setup). To complete the phase structure of the model we must explore it at non-zero chemical potential. This would require introducing a non-vanishing charge density by exciting additional components of the worldvolume gauge field.

\section{Acknowledgments}

We thank Yago Bea and Johanna Erdmenger for discussions and Javier Mas for collaboration at the initial stages of this work. We are specially grateful to Veselin Filev for his comments and help. N. J. and A. V. R. are funded by the Spanish grant FPA2011-22594, by the Consolider-Ingenio 2010 Programme CPAN (CSD2007-00042), by Xunta de Galicia (GRC2013-024), and by FEDER. N. J. is also supported by the Juan de la Cierva program. D. Z. is funded by the FCT fellowship SFRH/BPD/62888/2009. Centro de Física do Porto is partially funded by FCT through the projects CERN/FP/116358/2010 and PTDC/FIS/099293/2008.

\section{A Zero temperature dictionary}

The relation between the mass and the parameter $\tilde{m}_{0}$ has been worked out in detail in section 2.5. In this appendix we work out the dictionary for the condensate at $T=0$, which we will denote by $\left\langle\mathcal{O}_{q}\right\rangle_{0}$. A similar analysis at non-zero temperature was presented in appendix D of [19]. For simplicity, in this appendix we use units in which $\alpha^{\prime}=1$.

Let $\mu_{q}^{0}$ be the bare quark mass at zero temperature, whose explicit expression in terms of $\tilde{m}_{0}$ and $B$ has been derived in section 2.5 (eq. (2.46)). The expectation value $\left\langle\mathcal{O}_{q}\right\rangle_{0}$ is 
obtained as the derivative with respect to $\mu_{q}^{0}$ of the zero temperature free energy:

$$
\left\langle\mathcal{O}_{q}\right\rangle_{0}=\frac{\partial F}{\partial \mu_{q}^{0}} .
$$

To compute the derivative in (A.1) we apply the chain rule:

$$
\frac{\partial F}{\partial \mu_{q}^{0}}=\frac{\partial F}{\partial \tilde{m}_{0}} \frac{\partial \tilde{m}_{0}}{\partial \mu_{q}^{0}},
$$

and use [19]:

$$
\frac{\partial F}{\partial \tilde{m}_{0}}=-\frac{3-2 b}{b^{2}} B^{\frac{3}{2}} \tilde{c}_{0} \mathcal{N}_{r}
$$

where $\tilde{c}_{0}=B^{\frac{b-3}{2}} c_{0}$. We get:

$$
\left\langle\mathcal{O}_{q}\right\rangle_{0}=-\frac{3-2 b}{b^{2}} B^{\frac{3}{2}} \tilde{c}_{0} \frac{\tilde{m}_{0}}{\mu_{q}^{0}} \mathcal{N}_{r} .
$$

Using (2.46) and the expression of $\mathcal{N}_{r}$, we find:

$$
\frac{\tilde{m}_{0}}{\mu_{q}^{0}} \mathcal{N}_{r}=\frac{(2-b) b^{2} \sigma}{4 \pi} N B^{-\frac{1}{2}}
$$

Therefore, we have the following relation between $\left\langle\mathcal{O}_{q}\right\rangle_{0}$ and $\tilde{c}_{0}$ :

$$
-\frac{\left\langle\mathcal{O}_{q}\right\rangle_{0}}{N}=\frac{(3-2 b)(2-b) \sigma}{4 \pi} B \tilde{c}_{0}
$$

which, after including the appropriate power of $\alpha^{\prime}$, coincides with the expression written in (2.47). Let us finally write (A.6) in terms of the physical magnetic field $B_{\text {phys }}$ given by $(2.15)$. We find

$$
-\frac{\left\langle\mathcal{O}_{q}\right\rangle_{0}}{N}=\frac{(3-2 b)(2-b)}{4 \pi^{2} \sqrt{2}} \frac{B_{\text {phys }}}{\sqrt{\lambda}} \tilde{c}_{0}
$$

Open Access. This article is distributed under the terms of the Creative Commons Attribution License (CC-BY 4.0), which permits any use, distribution and reproduction in any medium, provided the original author(s) and source are credited.

\section{References}

[1] D. Kharzeev, K. Landsteiner, A. Schmitt and H. -U. Yee, Strongly Interacting Matter in Magnetic Fields, Lect. Notes Phys. 871 (2013) 1.

[2] K. Klimenko, Three-dimensional Gross-Neveu model at nonzero temperature and in an external magnetic field, Theor. Math. Phys. 90 (1992) 1 [Teor. Mat. Fiz. 90 (1992) 3] [INSPIRE].

[3] K. Klimenko, Three-dimensional Gross-Neveu model at nonzero temperature and in an external magnetic field, Z. Phys. C 54 (1992) 323 [InSPIRE].

[4] K. Klimenko, Three-dimensional Gross-Neveu model in an external magnetic field, Theor. Math. Phys. 89 (1992) 1161 [Teor. Mat. Fiz. 89 (1991) 211] [INSPIRE]. 
[5] V. Gusynin, V. Miransky and I. Shovkovy, Catalysis of dynamical flavor symmetry breaking by a magnetic field in (2+1)-dimensions, Phys. Rev. Lett. 73 (1994) 3499 [Erratum ibid. 76 (1996) 1005] [hep-ph/9405262] [INSPIRE].

[6] V. Gusynin, V. Miransky and I. Shovkovy, Dimensional reduction and dynamical chiral symmetry breaking by a magnetic field in (3+1)-dimensions, Phys. Lett. B 349 (1995) 477 [hep-ph/9412257] [INSPIRE].

[7] V. Miransky and I. Shovkovy, Magnetic catalysis and anisotropic confinement in QCD, Phys. Rev. D 66 (2002) 045006 [hep-ph/0205348] [INSPIRE].

[8] J.M. Maldacena, The Large- $N$ limit of superconformal field theories and supergravity, Adv. Theor. Math. Phys. 2 (1998) 231 [Int. J. Theor. Phys. 38 (1999) 1113] [hep-th/9711200] [INSPIRE].

[9] V.G. Filev, C.V. Johnson, R. Rashkov and K. Viswanathan, Flavoured large- $N$ gauge theory in an external magnetic field, JHEP 10 (2007) 019 [hep-th/0701001] [INSPIRE].

[10] V.G. Filev and R.C. Raskov, Magnetic Catalysis of Chiral Symmetry Breaking. A Holographic Prospective, Adv. High Energy Phys. 2010 (2010) 473206 [arXiv:1010.0444] [INSPIRE].

[11] O. Bergman, J. Erdmenger and G. Lifschytz, A Review of Magnetic Phenomena in Probe-Brane Holographic Matter, Lect. Notes Phys. 871 (2013) 591 [arXiv:1207.5953] [INSPIRE].

[12] C. Núñez, A. Paredes and A.V. Ramallo, Unquenched Flavor in the Gauge/Gravity Correspondence, Adv. High Energy Phys. 2010 (2010) 196714 [arXiv:1002.1088] [INSPIRE].

[13] V.G. Filev and D. Zoakos, Towards Unquenched Holographic Magnetic Catalysis, JHEP 08 (2011) 022 [arXiv:1106.1330] [INSPIRE].

[14] J. Erdmenger, V.G. Filev and D. Zoakos, Magnetic Catalysis with Massive Dynamical Flavours, JHEP 08 (2012) 004 [arXiv:1112.4807] [INSPIRE].

[15] O. Aharony, O. Bergman, D.L. Jafferis and J. Maldacena, N=6 superconformal Chern-Simons-matter theories, M2-branes and their gravity duals, JHEP 10 (2008) 091 [arXiv:0806.1218] [INSPIRE].

[16] S. Hohenegger and I. Kirsch, A Note on the holography of Chern-Simons matter theories with flavour, JHEP 04 (2009) 129 [arXiv: 0903.1730] [INSPIRE].

[17] D. Gaiotto and D.L. Jafferis, Notes on adding D6 branes wrapping $R p^{3}$ in $A d S_{4} \times C P^{3}$, JHEP 11 (2012) 015 [arXiv:0903.2175] [INSPIRE].

[18] E. Conde and A.V. Ramallo, On the gravity dual of Chern-Simons-matter theories with unquenched flavor, JHEP 07 (2011) 099 [arXiv: 1105.6045] [INSPIRE].

[19] N. Jokela, J. Mas, A.V. Ramallo and D. Zoakos, Thermodynamics of the brane in Chern-Simons matter theories with flavor, JHEP 02 (2013) 144 [arXiv:1211.0630] [INSPIRE].

[20] Y. Bea, E. Conde, N. Jokela and A.V. Ramallo, Unquenched massive flavors and flows in Chern-Simons matter theories, JHEP 12 (2013) 033 [arXiv:1309.4453] [INSPIRE].

[21] G. Veneziano, Some Aspects of a Unified Approach to Gauge, Dual and Gribov Theories, Nucl. Phys. B 117 (1976) 519 [InSPIRE].

[22] A. Karch and A. O'Bannon, Metallic AdS/CFT, JHEP 09 (2007) 024 [arXiv:0705.3870] [INSPIRE]. 
[23] O. Bergman, N. Jokela, G. Lifschytz and M. Lippert, Quantum Hall Effect in a Holographic Model, JHEP 10 (2010) 063 [arXiv: 1003.4965] [INSPIRE].

[24] O. Bergman, G. Lifschytz and M. Lippert, Magnetic properties of dense holographic QCD, Phys. Rev. D 79 (2009) 105024 [arXiv:0806.0366] [INSPIRE].

[25] N. Jokela, G. Lifschytz and M. Lippert, Magnetic effects in a holographic Fermi-like liquid, JHEP 05 (2012) 105 [arXiv: 1204.3914] [INSPIRE].

[26] D. Mateos, R.C. Myers and R.M. Thomson, Thermodynamics of the brane, JHEP 05 (2007) 067 [hep-th/0701132] [INSPIRE].

[27] V.G. Filev, Hot Defect Superconformal Field Theory in an External Magnetic Field, JHEP 11 (2009) 123 [arXiv:0910.0554] [INSPIRE].

[28] G. Bali, F. Bruckmann, G. Endrodi, Z. Fodor, S. Katz et al., QCD quark condensate in external magnetic fields, Phys. Rev. D 86 (2012) 071502 [arXiv:1206.4205] [INSPIRE].

[29] F. Preis, A. Rebhan and A. Schmitt, Inverse magnetic catalysis in dense holographic matter, JHEP 03 (2011) 033 [arXiv: 1012.4785] [INSPIRE]. 